\title{
Probing local order in multiferroics by transmission electron microscopy
}

\author{
${ }^{1}$ Electron Microscopy Center, Swiss Federal Laboratories for Materials Science and Technology, Empa, Dübendorf, Zurich 8600 , \\ Switzerland, E-mail: Marco.Campanini@empa.ch, Rolf.Erni@empa.ch, marta.rossell@empa.ch
}

\begin{abstract}
:
The ongoing trend toward miniaturization has led to an increased interest in the magnetoelectric effect, which could yield entirely new device concepts, such as electric field-controlled magnetic data storage. As a result, much work is being devoted to developing new robust room temperature (RT) multiferroic materials that combine ferromagnetism and ferroelectricity. However, the development of new multiferroic devices has proved unexpectedly challenging. Thus, a better understanding of the properties of multiferroic thin films and the relation with their microstructure is required to help drive multiferroic devices toward technological application. This review covers in a concise manner advanced analytical imaging methods based on (scanning) transmission electron microscopy which can potentially be used to characterize complex multiferroic materials. It consists of a first broad introduction to the topic followed by a section describing the so-called phase-contrast methods, which can be used to map the polar and magnetic order in magnetoelectric multiferroics at different spatial length scales down to atomic resolution. Section 3 is devoted to electron nanodiffraction methods. These methods allow measuring local strains, identifying crystal defects and determining crystal structures, and thus offer important possibilities for the detailed structural characterization of multiferroics in the ultrathin regime or inserted in multilayers or superlattice architectures. Thereafter, in Section 4, methods are discussed which allow for analyzing local strain, whereas in Section 5 methods are addressed which allow for measuring local polarization effects on a length scale of individual unit cells. Here, it is shown that the ferroelectric polarization can be indirectly determined from the atomic displacements measured in atomic resolution images. Finally, a brief outlook is given on newly established methods to probe the behavior of ferroelectric and magnetic domains and nanostructures during in situ heating/electrical biasing experiments. These in situ methods are just about at the launch of becoming increasingly popular, particularly in the field of magnetoelectric multiferroics, and shall contribute significantly to understanding the relationship between the domain dynamics of multiferroics and the specific microstructure of the films providing important guidance to design new devices and to predict and mitigate failures.
\end{abstract}

Keywords: (scanning) transmission electron microscopy, multiferroics, phase-contrast techniques, electron nanodiffraction, strain mapping, local ferroelectric polarization, in situ measurements

DOI: $10.1515 /$ psr-2019-0068

\section{Introduction}

Over the last decade, numerous experimental methods based on transmission electron microscopy have undergone quantum leaps. These abrupt advancements are mainly due to the significantly improved stability of microscope platforms and the availability of new electron optical devices. Aside from high performance spectrometers for electron energy-loss (EELS) and energy-dispersive X-ray spectroscopies (EDXS), fast and highly sensitive digital cameras based on CMOS technology have been introduced. These advanced cameras have replaced the inherently sluggish slow-scan CCD cameras which were not optimal, particularly for monitoring dynamics by in situ measurements. Moreover, novel detector geometries, electron monochromators and most notably aberration correctors, which can correct the intrinsic geometrical and ultimately also the chromatic aberrations of electromagnetic electron lenses, have significantly improved the information which can be gathered from atomic-resolution micrographs [1,2]. Most noteworthy consequences of these technical improvements are that true atomic resolution micrographs of heavy and light atoms can be recorded in transmission and scanning transmission electron microscopy (TEM/STEM) approaching or exceeding half an Ångström resolution. In addition, atomic potentials can be mapped with high signal-to-noise ratios and with minimal blurring enabling the determination of atomic positions with precision in the picometer regime. New detection strategies 
make it possible to map electrostatic and magnetic fields with unprecedented resolutions. And from an analytical point of view, the high-performance spectrometers mentioned above enable the detection of highly sensitive chemical signals as well as electronic structure information by EELS or EDX of individual atomic columns and even single atoms [3]. Materials, their atomic structure and chemistry, can thus be characterized down to the atomic level. The main advantage of these electron microscopy-based methods over other techniques is that information can be extracted locally, at defect sites such as dislocation cores, at phase and domain boundaries and at other irregularities. For averaged information, diffraction methods and other techniques might provide a more precise, in terms of its statistical significance, but a less detailed picture.

When it comes to the characterization of the polar order in multiferroic materials, the full advantage of all the technical achievements can be exploited. Precise derivation of atomic positions based on high-resolution micrographs enables local and quantitative measurements of strain states, the distortion of unit cells or the (re-)arrangement of atoms within these unit cells. This is particularly important as the ferroelectric properties are (often) coupled to the symmetry of the unit cell and the relative positions of the atoms therein. However, for magnetic order in multiferroics the situation becomes a bit more intricate as the lenses used in medium and high-voltage transmission electron microscopes $(60-300 \mathrm{kV})$ are made of magnetic fields, which in the objective lens (OL), i. e. the most important lens of the microscope, can reach values between about 1 and $2 \mathrm{~T}$ at normal operational conditions. Hence, the environmental magnetic field of the OL, wherein the specimen is immersed, prevents the study of the intrinsic domain structure of (ferro- and ferri-)magnetic materials. Operating the TEM with the OL turned off by using a lens either before or after the sample in order to focus the electron beam in STEM or TEM, respectively, allows for preserving the specimen's magnetic structure during the measurement, but comes at the expense of less resolution. These so-called Lorentz TEM or Lorentz STEM modi enable an image resolution in the range of typically $1 \mathrm{~nm}$ [4]. Though in general atomic resolution is not feasible in Lorentz modus, the resolution is still sufficient to derive critical information about the magnetic domain structure of the sample whose typical length scale typically exceeds the nanometer range.

So far, methods have been mentioned which improved the inspection of samples in the physical state it was prepared and transferred into the vacuum of the microscope column. Such retroactive measurements are important to learn about the basic states of matter but do not allow for finding out how the material had reached that state. Another branch of TEM applications, whose capabilities have started to flourish, covers so-called in situ or in operando measurements [2]. The goal of such measurements is to change the properties and/or the physical state of the sample in a controlled way in the microscope while the sample is under observation. One might argue that the small TEM samples, which are merely tens of nanometer thick, do not represent the state of matter as it adopts in a device. Nevertheless, such approaches allow for uncovering fundamental mechanisms, like, e. g. the change of the local atomic structure while temperature or electrical bias is varied or the moving mechanisms of domain walls and the identification of pinning centers. Though in situ transmission electron microscopy is not new at all, the recent success of these methods lies in the availability of special sample holders for MEMS (Micro-Electro-Mechanical Systems) chips upon which a small piece of the sample material, normally extracted and prepared by a focused ion beam (FIB) instrument, is mounted and contacted to the chip's electrodes. The advantages of the MEMS-based in situ technology over the conventional approach, i. e. where typically the state of disk-shaped samples of several millimeter diameter is being stimulated, lies in the fact that control and homogeneity over the physical state and the applied stimulus are enhanced. Moreover, because of the smaller volumes inspected in MEMS devices, the sample drift is minimal which is particularly important for heating experiments. In addition, cooling or heating rates can be achieved which are orders of magnitude higher than what is achievable by a conventional thermal system. Hence, aside from studying samples extracted from devices and test architectures, modern transmission electron microscopes go beyond simple imaging tools. The implementation of in situ techniques enabled by MEMS-based sample holders provides a physical test bench inside the microscope with the advantage that while the material is undergoing an induced transition, it can be inspected at a variety of length scales even down to the atomic scale where changes in the structure of individual unit cells can be observed.

While, of course, the study of ferromagnetic and ferroelectric materials, their defects and domains, is not new in transmission electron microscopy by using a variety of methods, such as CBED, conventional brightand dark-field transmission electron microscopy or, e. g. Lorentz TEM, in this review we would like to focus on (i) new high-resolution electron microscopy techniques which can be used to characterize magnetoelectric multiferroics and which can be used to learn about the local order parameters in these materials, (ii) on new experimental strategies of measuring the macroscopic electrostatic and magnetic fields and (iii) on the new possibilities of in situ or in operando measurements. This review thus targets on introducing and discussing newly established techniques, whose potential might not have yet been fully explored, using specific examples for illustration. 


\section{S/TEM techniques for field mapping}

In ferroic materials, the formation of an ordered state is usually associated with a symmetry breaking of the system [5]. Such symmetry breaking responsible for the ferroic order is conveniently characterized by an order parameter, which describes the degree of order of the microscopic entities relevant to define the ferroic state of the system. The most common examples of order parameters are the polarization $\mathrm{P}$ and the magnetization $\mathrm{M}$, which respectively describe the degree of order of the microscopic electric dipoles and spins within the material.

In multiferroic materials the combination of different symmetry breakings leads to the coupling of order parameters, allowing for modifying one ferroic state by the application of a conjugated impulse. Among multiferroic materials, magnetoelectric multiferroics are materials in which ordered states of spins and electric dipoles coexist and are coupled, in such a way that the application of an external electric field can change the magnetic properties of the material and vice versa [6]. The complex phenomenology of magnetoelectric materials arises as a consequence of their peculiar morphology and structure, which display a wide range of features at different length scales including polar and magnetic domains, domain walls, vortices, etc. The investigation of ferroelectric and ferromagnetic materials is thus very challenging, involving different length scales that range from the meso- to the nanoscale.

Phase-contrast techniques in transmission electron microscopy offer the unique advantage of high spatial resolution and direct sensitivity to electrostatic and magnetic fields [7-10], allowing a multi-scale investigation of multiferroic materials. The qualitative and quantitative estimation of the order parameters describing the ferroic state and their evolution during the transformations - e. g. from the disordered to the ordered phase - is of fundamental importance for understanding the material properties and interpret the material behavior under applied external stimuli.

Recently, phase-contrast techniques have been successfully employed - usually in combination with in situ cryogenic capabilities - in the investigation of novel spin structures (see DOI: 10.1515/PSR.2019.0016), including topological structures, such as magnetic skyrmions [11, 12], vortices and anti-vortices [13]. In this framework, phase-contrast techniques hold great promise for exploring dynamical magnetoelectric phenomena, as proven by state-of-the-art in situ experiments like e. g. [14, 15] and discussed in DOI: 10.1515/PSR.2019.0017.

This subsection focuses on the application of phase-contrast techniques in transmission electron microscopy in the investigation of the fundamental aspects of multiferroic materials and their static properties, with a special emphasis on the study of domain configurations, mesoscopic polarization, and atomic electric fields.

\subsection{The spatial resolution of S/TEM phase-contrast techniques}

The properties of multiferroic materials can be investigated in transmission electron microscopy by means of socalled phase-contrast techniques, i. e. electron holography, Lorentz microscopy, and differential phase-contrast STEM. In the case of ferroelectric specimens, such studies can be carried out at a length scale ranging from the nano to the atomic scale, while for magnetic specimens the constraints due to the geometry of the OL limit the resolution to the nanometer range.

In common transmission electron microscopes, the OL works in the immersive mode, i. e. the sample is inserted in between the lens pole pieces where the magnetic field has its maximum value (about $2 \mathrm{~T}$ ) [16]. The OL has a very important role in the image formation process in both TEM and STEM modes and its strong magnetic field allows proper electron focusing and the achievement of high-resolution imaging. Nevertheless, the strong magnetic field generated by the OL in the specimen plane is deleterious for the study of the nanoscale magnetism since it can induce a significant alteration of the magnetic state of the specimen during the experiments.

Therefore, phase-contrast techniques for the study of magnetic properties - either in TEM or STEM mode - have to be performed in field-free conditions and the corresponding operating mode of the microscope is called Lorentz mode. The field-free condition can be achieved by switching-off the OL and using a different focusing lens that is located behind or before the specimen plane in the case of TEM or STEM operation modes, respectively. In modern microscopes, the Lorentz lens or the condenser mini-lens are employed to perform this task. Due to the limited resolution typical of Lorentz mode, the phase-contrast analysis of magnetic specimens gives access to their mesoscale properties, i. e. the magnetic domain configuration, the domain walls shape and geometry, topological charges (e. g. skyrmions), the stray fields, etc. On the contrary, the investigation of ferroelectrics is not affected by this resolution limitation. The polar properties can be investigated at nanometer resolution - in order to study the local ferroelectric order, the domain wall nature, etc. - or at atomic resolution. Aberration-corrected STEM holds great potential for studying the electric fields at atomic resolution, making accessible a direct comparison between the atomistic structure of the material and its functional properties. 
Here, it is worth mentioning that recently a new approach has been proposed in order to perform electron holography and Lorentz microscopy experiments on magnetic specimens without switching off the OL [17]. The method relies on compensating the magnetic fields generated by the upper and lower pole pieces at the specimen plane, by producing two equal magnetic fields but with an opposite sign. This special condition shall warrant a field-free environment for the sample without losing the resolving power provided by the OL, making this method very promising for the future development of magnetic field imaging with sub-nanometer resolution.

\subsection{The electron beam sensitivity to electrostatic and magnetic fields}

On transmitting an electron transparent sample, the illuminating electron wave undergoes both a change in amplitude and phase. While the change in amplitude is at the core of so-called amplitude contrast methods which are, e. g. exploited in bright-field or dark-field imaging where a particular Bragg diffracted beam is selected to form the image, the interaction of the electron beam with electrostatic and magnetic fields induces a change in the phase of the electron wavefunction - the Aharonov-Bohm effect [18] - given by:

$$
\phi(x, y)=\phi_{\mathrm{e}}+\phi_{\mathrm{m}}=\sigma \int_{-\infty}^{+\infty} \mathrm{V}(\mathrm{x}, \mathrm{y}, \mathrm{z}) \mathrm{dz}-\frac{\mathrm{e}}{\hbar} \int_{-\infty}^{+\infty} \mathrm{A}_{\mathrm{z}}(\mathrm{x}, \mathrm{y}, \mathrm{z}) \mathrm{dz}
$$

where $\sigma$ is the interaction parameter equal to $\sigma=\lambda \mathrm{me} /\left(2 \pi \hbar^{2}\right), \hbar$ the reduced Planck constant, V the electrostatic potential, and $\mathrm{A}_{\mathrm{z}}$ the component of the magnetic vector potential parallel to the electron beam propagation direction. In the interaction constant, $\lambda$ is the relativistic electron wavelength (that depends on the electron accelerating voltage), $\mathrm{m}$ is the electron relativistic mass, and e is the elementary charge. The integrals in eq. (1) are calculated along the electron trajectory, assuming the electron source and the detector plane at infinite distances from the specimen. In the following description, it is assumed that no other external electrostatic and magnetic fields exist, except for the one due to the specimen.

In the case the specimen does not display any ordered (ferroelectric/ferromagnetic) state, the only contribution to the phase shift of the electron beam is the one due to the mean inner electrostatic potential $\left(\mathrm{V}_{\text {mip }}\right)$ of the material and can be expressed as:

$$
\phi_{\mathrm{e}}(\mathrm{x}, \mathrm{y})=\sigma \mathrm{V}_{\text {mip }} \mathrm{t}(\mathrm{x}, \mathrm{y})
$$

with $t(x, y)$ the sample thickness at the lateral position $(x, y)$. The mean inner potential $V_{\text {mip }}$ is a materialdependent property that is defined as the volume average of the atomic electrostatic potentials of the specimen; accordingly, $\mathrm{V}_{\text {mip }}$ depends on the local composition, the density, and ionicity of the specimen [19].

In the case of non-ferroic specimens with a uniform composition $\left(\mathrm{V}_{\text {mip }}=\right.$ const $)$, the electrostatic phase shift is directly related to the thickness of the specimen. The mean inner potential term can thus be employed to estimate the sample thickness. On the other hand, in the case of ferroic specimens, the mean inner potential contribution needs to be subtracted to the total phase shift to investigate the properties of the ordered state. Strong phase changes due to thickness variations (as it occurs, for example, at the edges of the specimen) are undesirable and can lead to a misinterpretation of the experiments, especially in low-dimensional systems like nanoparticles (NPs), nanowires (NWs) and thin films.

In ferroelectric specimens, the polarization is responsible for an additional contribution to the electrostatic phase shift - that we will here call $\phi_{\mathrm{P}}$ - which expresses the mesoscopic phase modulation of the electron wave due to the ferroelectric polarization. This contribution can be written as [20]:

$$
\phi_{\mathrm{P}}(\mathrm{x}, \mathrm{y})=\frac{\sigma}{\varepsilon} \int_{0}^{\mathrm{t}}\left[\int_{0}^{(\mathrm{x}, \mathrm{y})} \mathbf{P}_{\perp}\left(\mathrm{x}^{\prime}, \mathrm{y}^{\prime}\right) \mathrm{dx}^{\prime} \mathrm{dy}^{\prime}\right] \mathrm{dz}
$$

With $\varepsilon=\varepsilon_{0} \cdot \varepsilon_{\mathrm{r}}$ the dielectric constant given by the product of the vacuum $\left(\varepsilon_{0}\right)$ and the relative $\left(\varepsilon_{\mathrm{r}}\right)$ permittivity and $\mathbf{P}_{\perp}$ the component of the polarization orthogonal to the electron beam propagation direction. The use of the relative permittivity in eq. (3) is meant to include the depolarizing effect of free charges which reduce the polarization value of the system. Assuming a constant polarization across the thickness $t$, we obtain: 


$$
\phi_{\mathrm{P}}(\mathrm{x}, \mathrm{y})=\frac{\sigma \mathrm{t}(\mathrm{x}, \mathrm{y})}{\varepsilon}\left[\int_{0}^{(\mathrm{x}, \mathrm{y})} \mathrm{P}_{\perp}\left(\mathrm{x}^{\prime}, \mathrm{y}^{\prime}\right) \mathrm{dx}^{\prime} \mathrm{dy}^{\prime}\right]
$$

Therefore, in the case of a specimen with a constant thickness $(t(x, y)=$ const) and uniform composition $\left(\mathrm{V}_{\text {mip }}=\right.$ const $)$, the electrostatic phase shift becomes:

$$
\phi_{\mathrm{e}}(\mathrm{x}, \mathrm{y})=\sigma \mathrm{V}_{\mathrm{mip}} \mathrm{t}+\phi_{\mathrm{P}}(\mathrm{x}, \mathrm{y})=\phi_{\mathrm{P}}(\mathrm{x}, \mathrm{y})+\mathrm{const}
$$

The in-plane components $\left[\mathrm{P}_{\mathrm{x}}^{\perp}(\mathrm{x}, \mathrm{y}), \mathrm{P}_{\mathrm{y}}^{\perp}(\mathrm{x}, \mathrm{y})\right]$ of the projected polarization can thus be retrieved from the phase image by means of the following expression:

$$
\left[\mathrm{P}_{\mathrm{x}}^{\perp}(\mathrm{x}, \mathrm{y}), \mathrm{P}_{\mathrm{y}}^{\perp}(\mathrm{x}, \mathrm{y})\right]=\frac{\varepsilon}{\sigma \mathrm{t}} \nabla_{\mathrm{xy}} \phi_{\mathrm{P}}(\mathrm{x}, \mathrm{y}) \quad \stackrel{\substack{\mathrm{V} \\ \mathrm{mip} \\ \mathrm{t}=\text { const }}}{\rightarrow} \frac{\varepsilon}{\sigma t} \nabla_{\mathrm{xy}} \phi_{\mathrm{e}}(\mathrm{x}, \mathrm{y})
$$

where the symbol $\nabla_{x y}$ denotes the in-plane gradient operator given by $(\partial / \partial x, \partial / \partial y)$.

As mentioned above, in specimens of inhomogeneous thickness and/or variable composition the local changes in the mean inner potential can hamper the proper investigation of the specimen polarization. In such cases, the expression marked by the asterisk (*) in eq. (6) does not hold and the ferroelectric contribution to the phase has to be isolated.

In the case the specimen displays an ordered magnetic state, the magnetic contribution to the phase shift $\phi_{\mathrm{m}}$ also needs to be considered. It can be shown that the magnetic phase shift is related to the magnetic flux (see scheme in Figure 1) by calculating the difference between $\phi_{\mathrm{m}}$ values at two arbitrary points $\left(\mathrm{x}_{1}, \mathrm{x}_{1}\right)$ and $\left(\mathrm{x}_{2}, \mathrm{x}_{2}\right)$ in the phase image [10]:

$$
\Delta \phi_{\mathrm{m}}=\phi_{\mathrm{m}}\left(\mathrm{x}_{1}, \mathrm{y}_{1}\right)-\phi_{\mathrm{m}}\left(\mathrm{x}_{2}, \mathrm{y}_{2}\right)=-\frac{\mathrm{e}}{\hbar} \int_{-\infty}^{+\infty} \mathrm{A}_{\mathrm{z}}\left(\mathrm{x}_{1}, \mathrm{y}_{1}, \mathrm{z}\right) \mathrm{dz}+\frac{\mathrm{e}}{\hbar} \int_{-\infty}^{+\infty} \mathrm{A}_{\mathrm{z}}\left(\mathrm{x}_{2}, \mathrm{y}_{2}, \mathrm{z}\right) \mathrm{dz}
$$

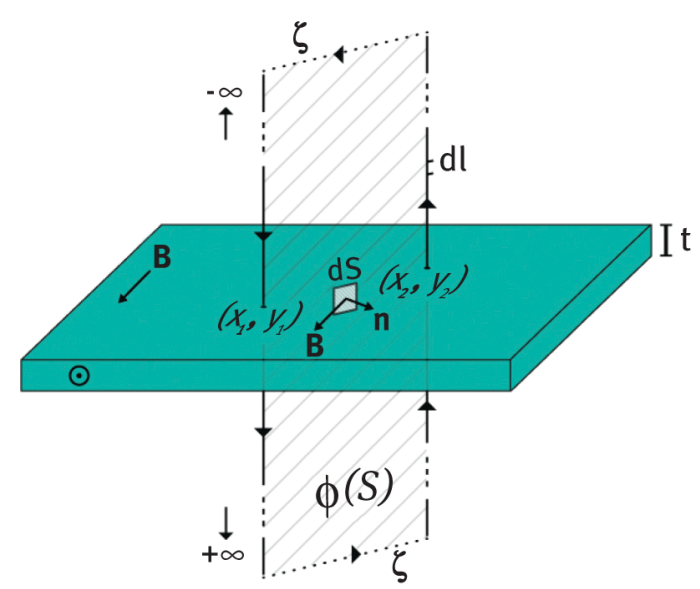

Figure 1: Schematic diagram of a uniformly magnetized thin film of constant thickness $t$. $\Phi$ is the magnetic flux through the surface $S$, bounded to the electron trajectories in $\left(x_{1}, y_{1}\right)$ and $\left(x_{2}, y_{2}\right)$.

Assuming that the magnetic potential generated by the specimen vanishes at infinite distance and no other transversal magnetic fields exist along the trajectories of the electrons, the amount given in eq. (7) can be alternatively expressed by a loop integral over a rectangular path, constituted by the two parallel electron trajectories - in the positions $\left(\mathrm{x}_{1}, \mathrm{y}_{1}\right)$ and $\left(\mathrm{x}_{2}, \mathrm{y}_{2}\right)$ - merged at infinite distances above and below the specimen plane by two segments $(\zeta)$ perpendicular to the trajectories themselves. The loop integral can be written as:

$$
\Delta \phi_{m}=-\frac{\mathrm{e}}{\hbar} \oint \mathbf{A} \cdot \mathrm{dl}
$$


Using the Stokes' theorem, the loop integral of the magnetic potential can be expressed by the magnetic flux $(\Phi)$ through the region of space (S) enclosed by the two electron trajectories $\left(\mathrm{x}_{1}, \mathrm{y}_{1}\right)$ and $\left(\mathrm{x}_{2}, \mathrm{y}_{2}\right)$ :

$$
\Delta \phi_{\mathrm{m}}=\frac{e}{\hbar} \iint \mathbf{B} \cdot \hat{\mathbf{n}} \mathrm{ds}=\frac{\pi}{\phi_{0}} \Phi(\mathrm{S})
$$

where $\phi_{0}=\mathrm{h} / 2 \mathrm{e}=2.07 \cdot 10^{-15} \mathrm{~T} \mathrm{~m}^{2}$ represents the "flux quantum" [10].

From the magnetic contribution to the phase shift, we can obtain the in-plane components of the magnetic induction $\left[\mathrm{B}_{\mathrm{x}}^{\mathrm{P}}(\mathrm{x}, \mathrm{y}), \mathrm{B}_{\mathrm{y}}^{\mathrm{P}}(\mathrm{x}, \mathrm{y})\right]$ by calculating the gradient of $\phi_{\mathrm{m}}[10,21]$ :

$$
\begin{aligned}
& \frac{\partial \phi_{m}(x, y)}{\partial x}=-\frac{e}{\hbar} \int \frac{\partial A_{z}(x, y, z)}{\partial x} d z=+\frac{e}{\hbar} B_{y}^{P}(x, y) \\
& \frac{\partial \phi_{m}(x, y)}{\partial y}=-\frac{e}{\hbar} \int \frac{\partial A_{z}(x, y, z)}{\partial y} d z=-\frac{e}{\hbar} B_{x}^{P}(x, y)
\end{aligned}
$$

where we replaced the partial derivatives of $A_{z}$ using the expressions obtained by the magnetic potential definition:

$$
B_{x}=\frac{\partial A_{z}(x, y)}{\partial y}, \quad B_{y}=-\frac{\partial A_{z}(x, y)}{\partial x}
$$

The formulas given in eq. (10) highlight the direct relationship between the magnetic phase shift and the inplane components of the magnetic induction, which can be summarized as:

$$
\left[\mathrm{B}_{\mathrm{y}}^{\mathrm{P}}(\mathrm{x}, \mathrm{y}),-\mathrm{B}_{\mathrm{x}}^{\mathrm{P}}(\mathrm{x}, \mathrm{y})\right]=\frac{\hbar}{\mathrm{e}} \nabla \phi_{\mathrm{m}}(\mathrm{x}, \mathrm{y})
$$

\subsection{Phase-contrast techniques in S/TEM}

As previously described, the phase of the electron wave carries very useful information about the fields in the specimen. In conventional imaging techniques, however, we simply record an intensity distribution by collecting the electrons that impinge on a pixelated camera in the image plane (in TEM mode) or by integrating the scattered electrons over a certain angular range in the conjugated, i. e. diffraction, plane (in STEM mode). Unfortunately, in conventional imaging all the information directly related to the phase of the electrons is lost, being all the techniques solely sensitive to the intensity distribution of the scattered electrons. In order to study the electrostatic and magnetic fields in the specimen, we need to retrieve the phase shift imparted to the electron beam by the interaction with the sample. The techniques that allow retrieving the phase of the electron wave from intensity measurements are called phase-contrast techniques [22].

In particular, two different approaches allow mapping the specimen fields through phase-contrast techniques. The first one consists in retrieving the phase of the electron wavefunction by a reconstruction approach and calculating the fields by differentiating - using eqs. (6) and (12) - the phase of the wavefunction. The most common methods to measure the electron phase are off-axis electron holography and focal series reconstruction. The second approach is based on the direct measurement of the fields through DPC methods, from which the phase can be reconstructed upon integration. In this second category, DPC STEM with segmented-annular or pixelated detectors is gathering nowadays growing attention for its great potential, offering the advantages of high spatial-resolution, fast recording and parallel acquisition of different signals.

In the following, the basic principles of off-axis electron holography and DPC STEM are discussed, providing the reader with the fundamental tools to understand their application to ferroic materials investigations.

\subsubsection{Off-axis electron holography}

The full electron wavefront - in its amplitude and phase - can be retrieved by means of off-axis electron holography. This method consists in superimposing one electron beam that interacts with the specimen with another 
beam that propagates into the vacuum and acts as a reference wave [24]. After superimposing the two beams, we can record the interferometric pattern in the detector plane. In general, splitting of the beam is realized by partially illuminating the sample, meaning that one part of the beam, i. e. the object wave, passes through the specimen, while the other part, i. e. the reference wave, passes through vacuum. The superposition of the object and reference wave, respectively - is performed using the electrostatic biprism (also called Möllenstedt biprism [25]) placed behind the specimen plane. The superposition results in an interference pattern observable in the detector plane. A scheme of the electro-optical configuration commonly employed for electron holography experiments is sketched in Figure 2(a).
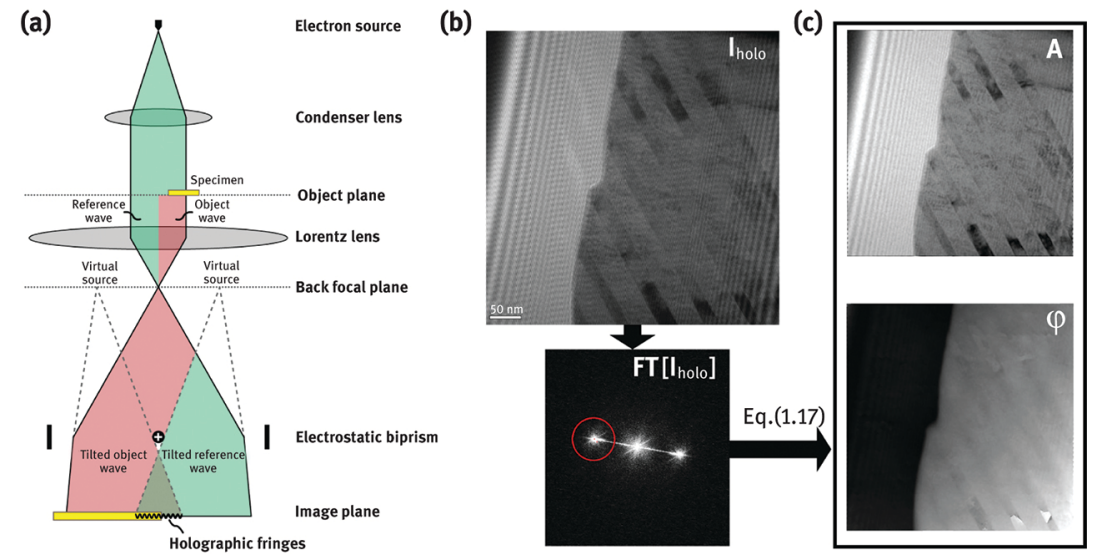

Figure 2: (a) Scheme of the conventional electron-optical configuration used for electron holography experiments (adapted from [23]). (b) Example of phase reconstruction from an electron holography experiment. One sideband is selected for the reconstruction of the amplitude and phase (c) through inverse Fourier transform operations.

We can write the electron wavefunction in the image plane as:

$$
\psi_{\mathrm{i}}(\mathbf{r})=\mathrm{A}_{\mathrm{i}}(\mathbf{r}) \mathrm{e}^{\mathrm{j} \phi_{\mathrm{i}}(\mathbf{r})}
$$

where $A_{i}$ and $\phi_{i}$ are respectively the electron wavefunction amplitude and phase in the image plane and $j$ is the imaginary unit. The recorded intensity in the detector plane is thus given by:

$$
\mathrm{I}(\mathbf{r})=\left|\mathrm{A}_{\mathrm{i}}(\mathbf{r})\right|^{2}
$$

As clearly visible in eq. (14), the information regarding the electron phase is lost in conventional imaging, as the intensity distribution is solely related to the wavefunction amplitude.

In electron holography, we perform an interferometric recording that allows for solving the phase problem. In fact, if we calculate the intensity in the image plane after superimposing the reference and object beams, we obtain:

$$
\mathrm{I}_{\text {holo }}(\mathbf{r})=\left|\psi_{\mathrm{i}}(\mathbf{r})+\mathrm{e}^{2 \pi \mathrm{j} \mathbf{q}_{\mathbf{T}} \cdot \mathrm{r}}\right|^{2}=1+\mathrm{A}_{\mathrm{i}}^{2}(\mathbf{r})+2 \mathrm{~A}_{\mathrm{i}}(\mathbf{r}) \cos \left[2 \pi \mathrm{j} \mathbf{q}_{\mathbf{T}} \cdot \mathbf{r}+\phi_{\mathrm{i}}\right]
$$

where $\mathbf{q}_{\mathrm{T}}$ is the wave-vector of the tilted reference beam. Eq. (15) shows that the intensity of an electron hologram is composed of three contributions, which are the unitary intensity of the reference image, the intensity of the image of the specimen, a modulated signal (given by the cosine term) that consists of interference fringes whose intensity maxima correspond to areas where the phase is the same for the object and reference beam. As an example, an electron hologram is shown in Figure 2(b).

The phase information is thus encoded in the intensity modulation appearing in the image and can be reconstructed by means of Fourier methods. In particular, taking the Fourier transform (FT) of the electron hologram, we obtain:

$$
\mathrm{FT}\left[\mathrm{I}_{\text {holo }}(\mathbf{r})\right]=\delta(\mathbf{q})+\operatorname{FT}\left[\mathrm{A}_{\mathrm{i}}{ }^{2}(\mathbf{r})\right]+\underbrace{\delta\left(\mathbf{q}+\mathbf{q}_{\mathbf{T}}\right) \otimes \mathrm{FT}\left[\mathrm{A}_{\mathrm{i}}(\mathbf{r}) \mathrm{e}^{\mathrm{j} \phi_{\mathrm{i}}(\mathbf{r})}\right]}_{(1)}+\underbrace{\delta\left(\mathbf{q}-\mathbf{q}_{\mathbf{T}}\right) \otimes \operatorname{FT}\left[\mathrm{A}_{\mathrm{i}}(\mathbf{r}) \mathrm{e}^{-\mathrm{j} \phi_{\mathrm{im}}(\mathbf{r})}\right]}_{(2)}
$$

The FT of the hologram is composed of two peaks centered at the origin of the reciprocal space - that correspond respectively to the FT of the reference and the FT of the image of the sample - and two peaks centered at $\mathbf{q}= \pm \mathbf{q}_{\mathbf{T}}$ 
(see Figure 2(c)). These latter two peaks, called sidebands and marked by (1) and (2) in eq. (16), are equal to the FT of the electron wavefunction and its complex conjugated, respectively. The amplitude and phase can finally be restored from one of the two isolated sidebands by shifting it to the center of the image and computing the inverse FT [10, 26]. The amplitude and phase are thus given by:

$$
\mathrm{A}_{\mathrm{i}}(\mathbf{r})=\left\{\operatorname{Re}\left[\psi_{\mathrm{i}}(\mathbf{r})\right]+\operatorname{Im}\left[\psi_{\mathrm{i}}(\mathbf{r})\right]\right\}^{\frac{1}{2}}, \phi_{\mathrm{i}}(\mathbf{r})=\tan ^{-1}\left\{\frac{\operatorname{Im}\left[\psi_{\mathrm{i}}(\mathbf{r})\right]}{\operatorname{Re}\left[\psi_{\mathrm{i}}(\mathbf{r})\right]}\right\}
$$

where we denoted the real and imaginary parts of the complex wavefunction with $R e$ and Im, respectively.

In the holography experiment, the spatial and phase resolution of the hologram are respectively related to the spacing and contrast of the fringes, which can be tuned by varying several parameters including the biprism voltage and the magnification [24,27]. Due to the opposite dependence of the contrast and spacing of the fringes on the biprism voltage, the experimental settings need to be properly chosen to achieve the desired spatial and phase resolutions. For the technical details about the optimal conditions (i. e. interference fringe spacing and signal-to-noise considerations) for the acquisition of electron holograms the reader is referred to more specific literature (see, e. g. [10, 28-32]).

In summary, electron holography experiments can be performed either at:

i. medium-resolution, for the investigation of the polar and magnetic properties of specimens. In this mode, the OL is switched off and the Lorentz lens is used instead to form the image. The achievable spatial resolution is limited by the aberration of the Lorentz lens and is usually in the nanometer range [23, 33].

ii. high-resolution, making use of the OL. This technique has been commonly used to improve the resolution of a high-resolution TEM image by the use of phase plates to correct for microscope lens aberrations [34-36].

However, it is important to recall that the investigation of magnetic materials has to be performed by mediumresolution electron-holography in Lorentz modus, which guarantees a field-free condition in the specimen plane, at the expense of the spatial resolution of the reconstructed phase.

Electron holography enables the retrieval of the total phase shift induced by the specimen to the electron beam. As previously discussed, the polar and magnetic properties of the specimen contribute to this phase with two different terms, which need to be separated. Depending on the terms that need to be addressed, this separation can be done by different methods. In the case of ferroelectric specimens, the ferroelectric phase shift can be separated from the mean inner potential contribution by performing the experiments respectively below and above the Curie temperature $\left(\mathrm{T}_{\mathrm{C}}\right)$. In fact, above $\mathrm{T}_{\mathrm{C}}$ the polar order is lost and the mean inner potential contribution is the only responsible for the phase shift. The phase difference of the two signals obtained respectively below and above $\mathrm{T}_{\mathrm{C}}$ permits thus to isolate the phase shift due to the polar properties of the material.

In ferromagnetic specimens, their net magnetization induces a magnetic term in the phase shift of the electron wavefunction. The electrostatic contribution must be removed from the reconstructed phase image in order to retrieve this contribution. The most common way to achieve this separation is by the time-reversal operation of the electron beam [37] that is performed by recording two holograms of the same region of the specimen in normal (upside) and reversal (downside) positions. The sum and the difference of the reconstructed phase images can then be used to recover the electrostatic and the magnetic contributions to the phase, respectively. An alternative approach involves performing an in situ magnetization reversal to detect the phase of two states of the specimen with opposite magnetization [38]. The addition of the two phase-images gives access to the mean inner potential contribution to the phase. Magnetization reversal can be performed by using either a magnetizing TEM specimen holder or the magnetic field of the OL (eventually in combination with specimen tilt). This second approach is only applicable if the magnetization of the specimen can be perfectly reversed.

\subsubsection{DPC STEM}

In DPC STEM a convergent electron probe is used to make a raster scan of the specimen and the variations in the CBED pattern are detected by means of a segmented annular detector or a pixelated camera [39]. The technique was firstly ideated in the '70s, thanks to the pioneering works by Rose [40] and Dekkers [41]. Nowadays, DPC STEM is experiencing a renaissance thanks to the spread of commercially available segmented detectors and fast pixelated cameras. DPC STEM is attracting indeed a continuously growing interest by the scientific community thanks to its great potential, which allows contemporary different imaging modes by simultaneous acquisition of various signals - like e. g. high-angle annular dark-field (HAADF) and bright-field (BF) - the acquisition of secondary signals for spectroscopic analysis like EELS and EDXS. Additionally, the sub-Ångström resolving power achievable thanks to the new generation of aberration-corrected microscopes [42] enables to carry out 
these investigations at atomic resolution. Up to date, DPC in the Lorentz STEM mode has been successfully employed to probe magnetic [15, 43-47] and electric fields [48-50] at the nanoscale, whereas DPC STEM at high-resolution has been employed to map electric fields at atomic resolution [51-54].

The DPC-STEM technique makes use of the deflection of the focused electron probe by a field in the sample to locally map this field. A scheme of the DPC principle is shown in Figure 3. If we describe classically, the propagation of the electron beam, the electrostatic and magnetic fields in the specimen plane induce a tilt of the electron probe in the specimen plane which leads to a shift of the bright field disk ("Ronchigram") in the diffraction plane. The DPC-STEM image is generated by performing a raster scan of the beam across the sample and recording for each scan position the off-centering of the transmitted disk. The variation of the disk position in the diffraction plane can be efficiently detected by a segmented detector. In particular, the off-centering of the disk induces an increase of the intensity impinging on certain detector segments at the expense of others, which are consequently less illuminated. The beam deflection angle can thus be estimated by measuring the differential contrast between opposite pairs of quadrants or - in a more generic segmented detector configuration, like e. g. the 16-quadrant configuration [57]- opposite sets of quadrants.

(a)

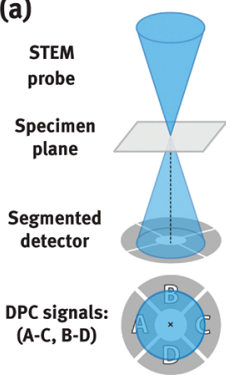

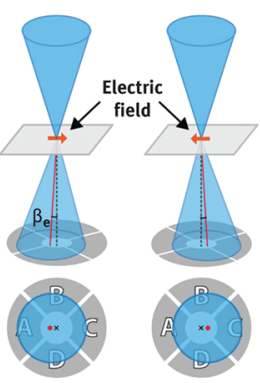

(b)

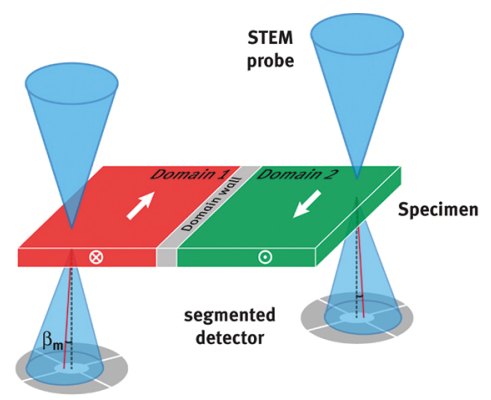

Figure 3: Scheme of the basic principles of DPC-STEM, performed using a segmented detector. (a) Electric and (b) magnetic field induced shift of the electron beam under the classical approximation for the electron propagation (adapted from $[55,56])$.

From the classic treatment of the electron propagation, it is possible to calculate the deflection of the electron beam due to the Lorentz force in presence of an electric or magnetic field in the specimen plane (respectively $\beta_{e}$ and $\left.\beta_{m}\right)[55,58]$ :

$$
\beta_{\mathrm{e}}\left(\mathbf{r}_{\mathbf{P}}\right)=\frac{\mathrm{e} \lambda}{\mathrm{hv}} \int_{0}^{\mathrm{t}} \mathbf{E}_{\perp}\left(\mathbf{r}_{\mathbf{P}}, z\right) \mathrm{dz}, \beta_{\mathrm{m}}\left(\mathbf{r}_{\mathbf{P}}\right)=-\frac{\mathrm{e} \lambda}{\mathrm{h}} \int_{0}^{\mathrm{t}} \mathbf{B}_{\perp}\left(\mathbf{r}_{\mathbf{P}}, \mathrm{z}\right) \times \mathrm{dz}
$$

where $v$ is the electron velocity and the integrals are performed on the specimen thickness $t\left(r_{P}\right)$ and $\mathbf{r}_{P}$ gives the position of the probe in the specimen plane. In the case of uniform electrostatic and magnetic fields, the relationships reduce to the handier forms:

$$
\beta_{\mathrm{e}}\left(\mathbf{r}_{\mathbf{P}}\right)=\frac{\mathrm{e} \lambda \mathrm{t}\left(\boldsymbol{r}_{\boldsymbol{P}}\right)}{\mathrm{hv}} \mathrm{E}_{\perp}\left(\mathbf{r}_{\mathbf{P}}\right), \beta_{\mathrm{m}}\left(\mathbf{r}_{\mathbf{P}}\right)=-\frac{\mathrm{e} \lambda \mathrm{t}\left(\boldsymbol{r}_{\boldsymbol{P}}\right)}{\mathrm{h}} \mathrm{B}_{\perp}\left(\mathbf{r}_{\mathbf{P}}\right)
$$

The deflections of the beam induced by electrostatic and magnetic fields can otherwise be calculated by an alternative approach. In particular, since DPC STEM does not measure the local phase shift but its gradient, eq. (19) can be derived by calculating the phase difference between electrons traveling along different trajectories and approximating the phase gradient with the finite difference among their phases [58].

It is interesting to note from eq. (19) that a local variation of the specimen thickness induces a change in the deflection angle. Therefore, the DPC-STEM technique can be used to directly measure the thickness variation of homogenous non-polar/non-magnetic specimens, where the specimen potential is solely given by the mean inner potential. In the case of ferroic materials, if the deflection angle caused by the effect that is to be measured is larger than the one caused by the thickness variations, the measurement will result with a good signal-tonoise ratio (the noise being the thickness variations at the area of interest) [58]. More generally, the reader should keep in mind that the thickness variations will generate some substructure in the signal of interest, which may lead to a misinterpretation of the experiments.

This simple model describing the electron beam deflection due to the Lorentz force allows for easily depicting the basic concepts behind the DPC-STEM technique. In reality, the quantification of fields in terms of a rigid shift of the Ronchigram can be inaccurate (Figure 4(a)) as a quantum mechanical treatment of the electron wave propagation is necessary (Figure 4(b)) [59,60]. From a quantum mechanics point of view, the electrostatic and 
magnetic fields in the specimen plane induce a variation on the distribution of probability to find an electron at a certain position in the detector plane or equivalently - due to the Fourier reciprocity between the specimen and the Fraunhofer diffraction planes - with a certain momentum. The expectation value of the momentum operator $\mathbf{p}$ is given by [60]:

$$
\left\langle\mathbf{p}_{\perp}\right\rangle\left(\mathbf{r}_{\mathbf{P}}\right)=\int\left\langle\psi_{\mathrm{D}}\left(\mathbf{p}, \mathbf{r}_{\mathbf{P}}\right)\left|\mathbf{p}_{\perp}\right| \psi_{\mathrm{D}}\left(\mathbf{p}, \mathbf{r}_{\mathbf{P}}\right)\right\rangle \mathrm{dp}_{\mathrm{x}} \mathrm{dp} \mathrm{p}_{\mathrm{y}}=\int \mathbf{p}_{\perp} \mathrm{I}\left(\mathbf{p}_{\perp}, \mathbf{r}_{\mathbf{P}}\right) \mathrm{dp} \mathrm{p}_{\mathrm{x}} \mathrm{dp}
$$

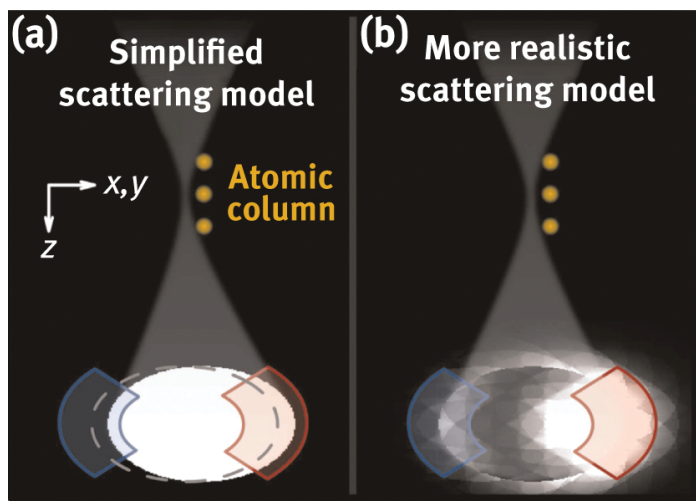

Figure 4: (a) Classical and (b) quantum-mechanical description of the effects of the electrostatic/magnetic fields on the DPC STEM signal. The quantum mechanical description includes dynamical effects that give rise to a complex redistribution of the intensity in the detector plane. Reproduced from [59].

which corresponds to the center of mass (CoM) of the Ronchigram in the detector plane. In the formula given in eq. (20), $\mathbf{r}_{\mathbf{P}}$ denotes the position of the convergent probe on the specimen, $\psi_{\mathrm{D}}\left(\mathbf{p}, \mathbf{r}_{\mathbf{P}}\right)$ is the electron wavefunction in the momentum space (correspondingly, in the detector plane), $\mathrm{I}\left(\mathbf{p}_{\perp}, \mathbf{r}_{\mathbf{P}}\right)$ the normalized intensity distribution in the diffraction plane. In the above expression, it is assumed that the beam intensity is constant over the specimen thickness.

If we aim at correlating the expectation value of the momentum in the detector plane with the fields in the specimen plane, we need to recall that in classical electrodynamics the Lorentz force is equal to the momentum transfer per time. According to Ehrenfest's theorem [61], we can write the quantum mechanical equivalent of the Lorentz force, which for the electrostatic field (the extension to the case of a magnetic field is straightforward) can be written as:

$$
\mathbf{E}_{\perp}\left(\mathbf{r}_{\mathbf{P}}\right)=-\left\langle\mathbf{p}_{\perp}\right\rangle\left(\mathbf{r}_{\mathbf{P}}\right) \cdot \frac{\mathrm{v}}{\mathrm{et}}
$$

where $\mathbf{E}_{\perp}$ is the electric field projection average along the electron beam direction, $\mathrm{v}$ is the electron velocity, e the elementary charge, and the specimen thickness. Although the rigid-shift model previously introduced does not accurately describe the electron propagation, the classical and the quantum mechanical treatment lead to similar results. In particular, from the quantum mechanical treatment, we obtain that the CoM of the Ronchigram - instead of the rigid shift of the beam - is linearly related to the projected local electric field in the sample (eq. (21)).

In order to make a proper estimation of the electric field, the expectation value of the momentum (as it is defined in eq. (20)) needs thus to be calculated. For this purpose, a pixel-array detector has to be used to record the full intensity distribution within the Ronchigram at each position of the STEM raster scan. This approach is commonly called 4D-STEM since it involves the acquisition of a two-dimensional (2D) diffraction image for each position of the raster scan - performed over a 2D grid of scan points across the region of interest CoM of the specimen. The acquisition of the entire Ronchigram also allows a posteriori integration of the scattered signal over different angular ranges of the CBED pattern to generate different signals, like e. g. bright-field (BF), annular bright-field (ABF), annular dark-field (ADF) [54, 62-64]. This very powerful feature makes 4D-STEM the most promising approach to combine differential phase-contrast and other imaging techniques in STEM mode, at the expense of a remarkable increase in the volume of generated data [65, 66]. Yet, one drawback of the currently existing 4D-STEM systems is the reduced scan speed which is given by the acquisition speed of the 2D camera. Current systems allow acquisition speeds of the order of 1000 frames per seconds [67,68], which is two to three orders of magnitude slower than common scan speeds in STEM.

As already described, a position-sensitive detector - i. e. a segmented detector - can be alternatively employed for the qualitative detection of the momentum CoM displacement by measuring the increase or decrease of signal in pairs of opposite quadrants. This well-established technology makes use of an integrating 
detector, with the advantages of faster scan speeds, high signal-to-noise ratio, live imaging, and it currently represents the most promising method for routine applications. Nevertheless, it appears evident from the quantum mechanical perspective that despite this technology is very practical, its application for quantitative analysis requires additional care because the use of a segmented detector only gives a qualitative approximation of the momentum CoM by roughly sampling the scattering distribution variation.

It must be noted that eq. (21) was obtained under the assumption that the intensity of the STEM probe does not change significantly along the propagation direction. A proper description of the quantum mechanical problem would involve the integration over the three-dimensional (3D) fields in the specimen weighted with the local intensity of the electron beam [60]. However, such an assumption is verified in these special relevant cases [69]:

i. under medium resolution imaging conditions. In this case, the STEM probe size is increased beyond the unit cell size and the sample can be oriented as to minimize the dynamical scattering. The channeling effects are negligible. Under these conditions, specimens can often be described as pure phase objects (phase object approximation, POA) [70].

ii. under high-resolution imaging conditions - i. e. namely, zone-axis orientation and evaluation of large scattering angles - for specimens in the thickness range for which the weak phase object approximation (WPOA) holds. Such range extends to a few nanometers in the case of specimens composed by light atomic species, whereas it reduces to a few Ångstrom for medium and heavy elements [69, 71]. In this thickness range, the broadening of the electron beam during its propagation can be neglected.

Since the WPOA is a questionable approximation for real specimens (with an exception for 2D materials, like e. g. graphene), the correct interpretation of atomic resolution DPC/4D-STEM data is neither trivial nor immediate. Additionally, the atomic electric fields vary very strongly over distances equal to fractions of the Bohr radius, which are smaller than the typical lateral size of aberration-corrected STEM probes. As a consequence, the interpretation of atomic resolution data requires comparison with simulations, as it is the case for other high-resolution S/TEM imaging techniques [59].

By fitting the CoM of the Ronchigram for each point in the raster scan, a projected electric field map can be obtained using eq. (21). Since the momentum transfer is linear with respect to the electric field, calculating its divergence we obtain:

$$
\operatorname{div}\left\langle\mathbf{p}_{\perp}\right\rangle=-\frac{e t}{v \varepsilon_{0}} \mathrm{e} \rho_{\perp}
$$

where $\mathrm{e} \rho_{\perp}$ is the overall projected charge density, given by the sum of densities of protons and electrons. On the other hand, by calculating the inverse gradient we obtain:

$$
\operatorname{grad}^{-1}\left\langle\mathbf{p}_{\perp}\right\rangle=\frac{e t}{v} V_{\perp}
$$

being $V_{\perp}$ the projected electrostatic potential. The eq. (23) shows that when the WPOA is valid - i. e. the phase shift of the electron wavefunction is $\phi \approx \sigma \mathrm{V}_{\perp}$ (see the conditions discussed previously) - the DPC and 4DSTEM are phase-contrast techniques in their integral perspective. Due to the finite size of the STEM probe, the experimentally measured electric field is the true electric field given in eq. (21) convolved with the electron probe, and similarly for the projected density of charge and projected potentials.

\subsubsection{Phase-contrast data analysis and representation}

As previously pointed out, the phase-contrast techniques allow for reconstructing the phase of the electron wavefunction and/or the projected in-plane fields. Therefore, the data obtained by phase-contrast techniques can be analyzed and plotted in different ways, depending on the features that need to be highlighted.

A very common way to represent the reconstructed phase, which gives visual information about the specimen field strength and direction, is by plotting the cosine of the phase [72]:

$$
\mathrm{I}(\mathbf{r})=\cos [\mathrm{n} \cdot \phi(\mathbf{r})]
$$

eventually multiplying the phase by an integer $\mathrm{n}$ as an amplification factor. In the case $n=1$, this representation gives the phase-contours for which the phase differs of $2 \pi$. The plot of phase-contours can be performed for 
both the electrostatic and magnetic phase contributions. Let us now analyze the meaning of the phase contours in both cases:

1. The contour lines obtained from the electrostatic contribution to the phase represent the electrostatic equipotential lines. The phase-contours are denser in the regions where the potential is rapidly changing and the electric field direction is normal to the contours. Assuming a constant specimen thickness or that the contribution of the mean inner potential has been properly subtracted, the phase-contours represent the equipotential lines for the ferroelectric phase-shift and the polarization.

2. The contour lines obtained for the magnetic phase shift represent the variation of $2 \pi$ of $\Delta \phi_{\mathrm{m}}$. From eq. (9) it follows that the magnetic flux between two points laying on consecutive contours is given by $2 \phi_{0}=414.10^{-15} \mathrm{~T} \mathrm{~m}^{2}$ [10]. In this case, from eq. (12) it follows that the magnetic induction vector is tangent to the phase contours.

Starting from the electric or magnetic field components - i. e. a 2D map for each vector-component-a vector plot of the corresponding field is possible. Such plots are usually presented by using an HSV (H: hue, S: saturation, V: value) color map, that is an alternative representation of the RGB color scheme. In this color map, the color is related to the direction and orientation of the field, and the hue is related to its magnitude.

\subsection{Investigations of electric and magnetic ordering in multiferroic materials}

Here, we briefly discuss the application of electron holography and differential phase-contrast STEM to the investigation of the local order in ferroelectric and ferromagnetic specimens through a few selected examples.

\subsubsection{Polar ordering in multiferroics}

Electron holography has been successfully employed to investigate the polar state of ferroelectric and multiferroic thin films and nanocrystals. A ferroelectric material $\left(\mathrm{BaTiO}_{3}\right)$ is used in the following cases as an instructive example, though the same analyses are valid for multiferroic materials.

Matsumoto and coworkers [73] applied medium-resolution electron holography to a thin film of $\mathrm{BaTiO}_{3}$ mapping the $90^{\circ}$ domain structure of the polar phase. In order to extract the phase shift due to ferroelectricity, the authors performed the holography experiments at RT - a condition in which the specimen was in the polar phase - and above the Curie temperature $\left(\mathrm{T}_{\mathrm{C}}\right)$ - i. e. without any polar order. Subtracting the phase shift obtained with the specimen heated above $\mathrm{T}_{\mathrm{C}}$ from that obtained at $\mathrm{RT}$ allows for isolating the contribution intrinsic to ferroelectricity from those originated from the mean inner potential - e. g. thickness variation - or from electron-beam-induced specimen charging. Figure 5(a) shows the reconstructed phase in a region with the $90^{\circ}$ ferroelectric domains. The reconstructed phase shows a weak contrast and faint stripes due to the ferroelectric domains are feebly visible, because of the thickness variation across the field of view. After subtracting the contribution of the mean inner potential, the phase features due to the polar domains are immediately visible (Figure 5(b-d)). 
(a)

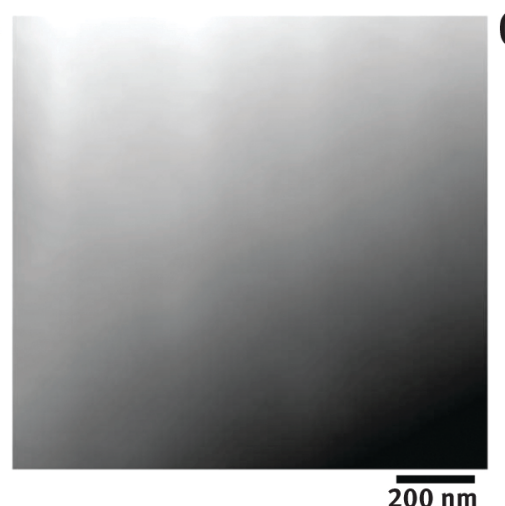

(c)

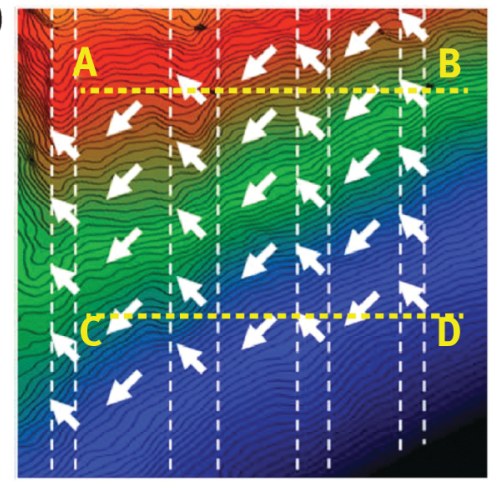

(b)

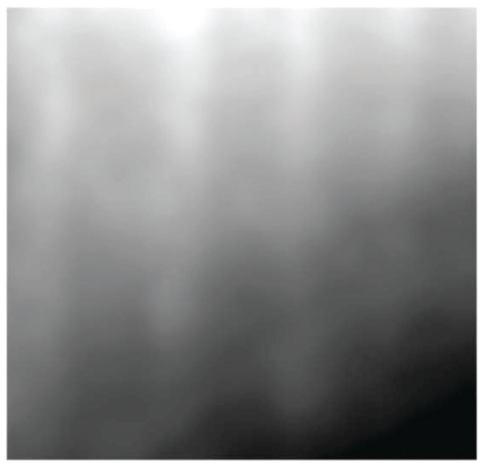

(d)
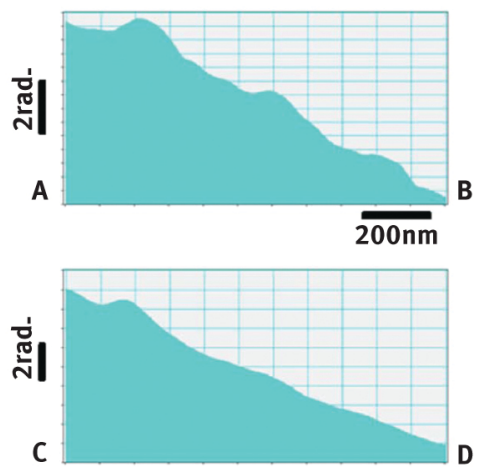

Figure 5: (a) Grayscale representation of the reconstructed phase of the $90^{\circ}$ domain structure in ferroelectric $\mathrm{BaTiO}_{3}$. (b) Difference between the reconstructed phases below and above $T_{C}$. (c) Color representation of the same phase difference overlaid with phase-contours and arrows representing the polarization. (d) Phase profiles along the segments AB and CD in (c). Reproduced from [73].

Polking and coworkers [74] applied medium-resolution electron holography to ferroelectric $\mathrm{BaTiO}_{3}$ nanocubes to investigate the effect of temperature and bias at the nanoscale. In particular, the $\mathrm{RT}$ polarized state of $\mathrm{BaTiO}_{3}$ nanocubes appears visible as a phase variation at the [001] facet of the nanocubes (Figure 6(a)). Such a phase change disappears by heating the specimen above $T_{C}$ (Figure 6(b)), where the contribution of the mean inner potential is the only one visible. Interestingly, a polar state (giving the same feature in the phase image) can be artificially induced by poling a nanocube with a W tip (Figure 4(c-f)). 
(a)

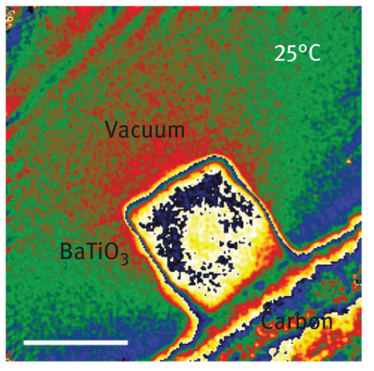

(c)

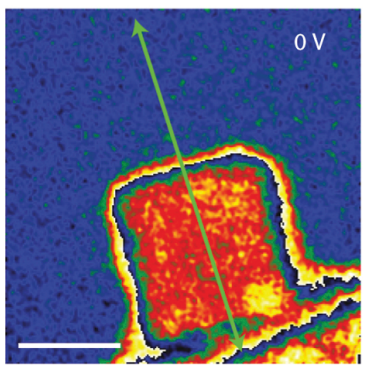

(e)

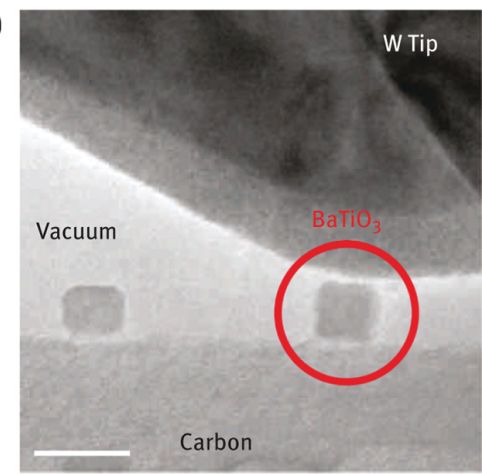

(b)

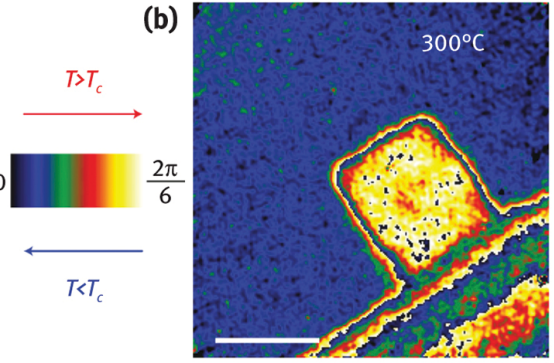

(d)
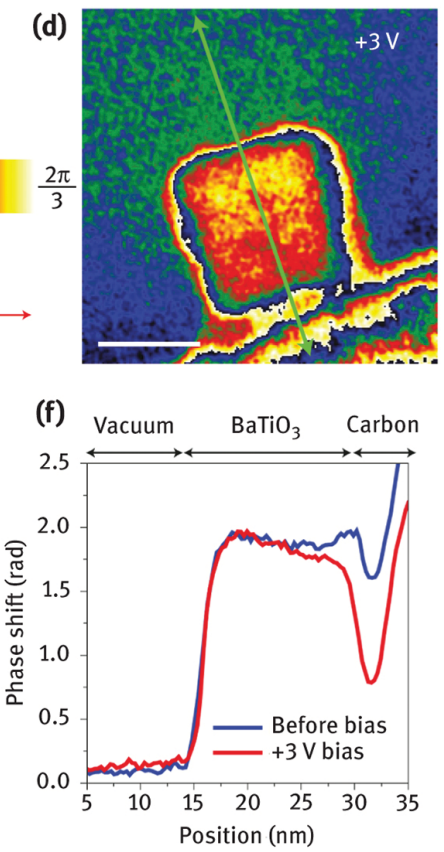

Figure 6: Phase images of a $\mathrm{BaTiO}_{3}$ nanocube below (a) and above (b) the Curie temperature (403 K). The phase variation in (a) highlights a polar state with the polarization oriented along the [001] direction. Phase image of a different nanocube before $(\mathrm{c})$ and after $(\mathrm{d})$ electrical poling $(+3 \mathrm{~V})$. The poling induces a net polarization as visible from the variation of phase along the [001] direction (green lines). (e) TEM image of the poling experiments. (f) Phase profiles along the green lines in (c) and (d). Reproduced from [74].

The nanoscale ferroelectric domain configuration in $\mathrm{BaTiO}_{3}$ thin films was successfully investigated also by medium-resolution DPC-STEM. Shibata and coworkers [51] applied the DPC technique performed using a four-quadrant segmented detector and measuring the differential signal between opposite pairs of detector segments. Figure 7(a and b) show the $\mathrm{BaTiO}_{3}$ unit cell distortion at the origin of the ferroelectric instability and the orientation of the specimen with respect to the segmented detector. The differential signal showed in Figure $7\left(\mathrm{c}\right.$ and $\mathrm{d}$ ) highlights $90^{\circ}$ ferroelectric domains (similar to the ones previously shown in Figure 5). 

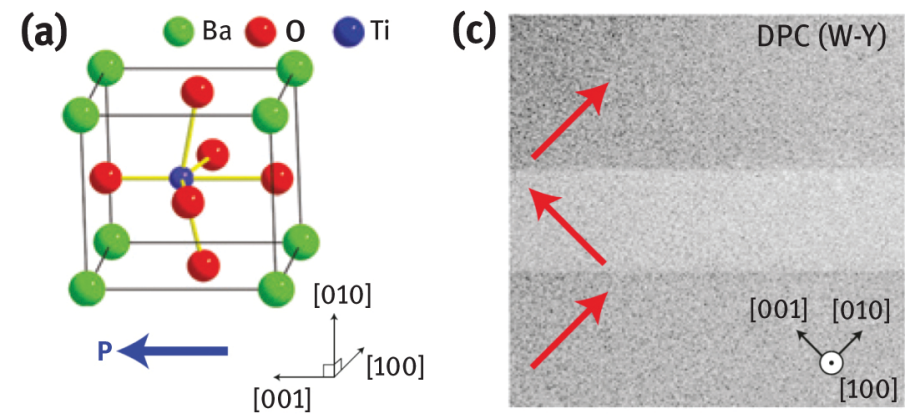

(b)

(d)
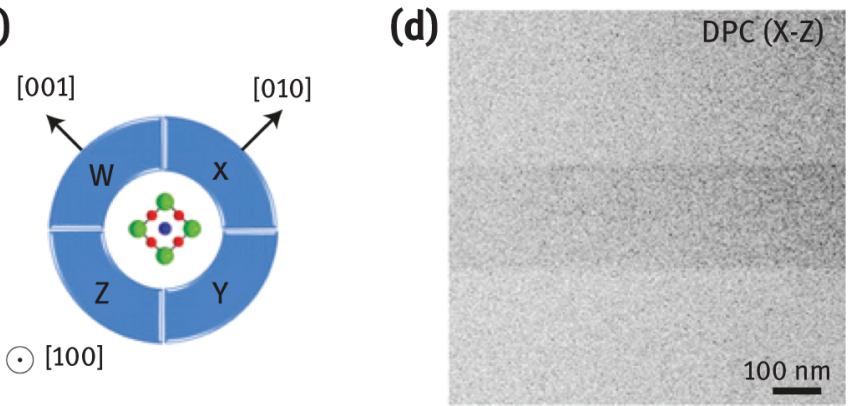

Figure 7: (a) Sketch of the $\mathrm{BaTiO}_{3}$ unit cell illustrating the structural distortion at the origin of the ferroelectric polarization. (b) Schematic representation of the unit cell orientation with respect to the detector quadrants. (c,d) Mediumresolution differential phase-contrast signals showing a change in the contrast corresponding to $90^{\circ}$ ferroelectric domains. The direction of the polarization is given by the red arrows in (c). Reproduced from [51].

As described in the previous section, DPC STEM has also been proven to be capable of atomic electric field mapping in high-resolution STEM mode. The correct interpretation of atomic electric field mapping is not straightforward, as it would require extensive use of image simulations to properly catch the dynamical effects which can strongly alter the momentum transfer in the reciprocal space. However, in ferroelectrics, the polarization typically varies very slowly (over distances comparable to the unit cell size) with respect to the atomic potentials, which are very strongly localized and peaked at the positions of the atomic nuclei. Since the polar field variation along the lateral size of the probe is very small, it will not affect significantly the dynamical scattering and its effect can be approximated as an additive low-frequency contribution superimposed to the strongly localized atomic fields.

As an example, we show the DPC-STEM analysis on a $\mathrm{SrRuO}_{3}(\mathrm{SRO}) / \mathrm{BaTiO}_{3}(\mathrm{BTO})$ bi-layer on a $\mathrm{SrTiO}_{3}$ substrate. Figure 8(a) shows a HAADF-STEM image of the specimen, in which a dislocation is visible in the upper BTO layer. The dislocation acts as a pinning center for a domain wall separating two differently oriented polar domains, as visible from the high-resolution images shown in the insets. Figure 8(b) shows a rotation map obtained by Geometric Phase Analysis (GPA) (a detailed explanation of GPA is given in Section 4) highlighting the dislocation position at the interface between the two upper SRO/BTO layers. In order to separate the strongly localized atomic electric field from the mesoscopic polarization which varies at the domain wall between the two differently oriented domains, we can perform DPC STEM experiments at atomic resolution and afterward perform Fourier filtering of the data (Figure 8(c)). By applying a band-pass filter (as shown in Figure $8(\mathrm{~d})$ ), we can create an image with the sole contribution of the wavevectors in the range $0.69-8.8 \mathrm{~nm}^{-1}$, which is equivalent to select the spatial frequencies in the range 1.45-11.2 $\AA$. The obtained reconstructed field does not contain any information about the mesoscopic polarization and only the field of the atomic columns is visible in the map. On the contrary, if we apply a low-pass filter (selecting the wavevectors smaller than $0.69 \mathrm{~nm}^{-1}$ ), we can retrieve the electric field due to the ferroelectric polarization. 

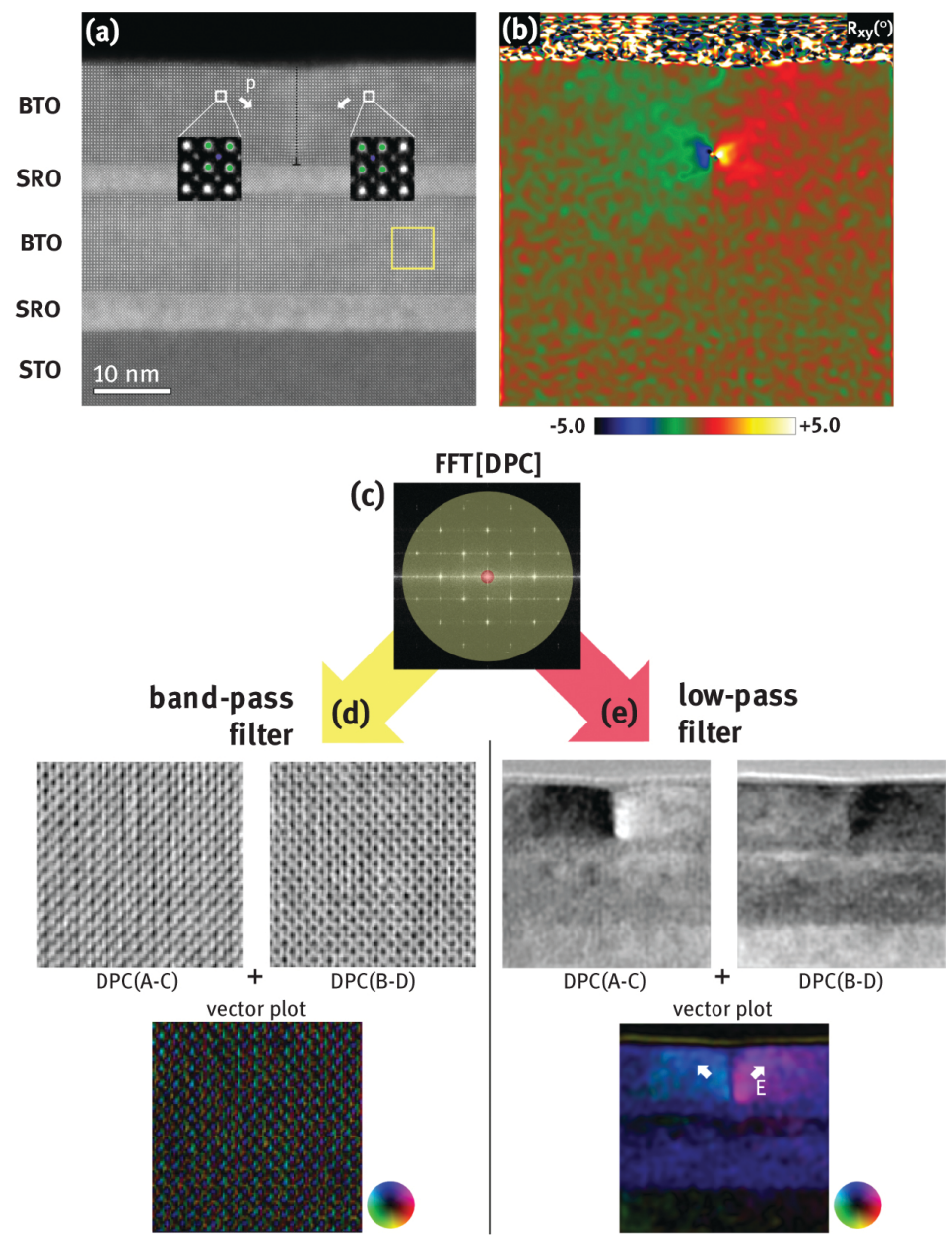

Figure 8: (a) HAADF-STEM image of a $\mathrm{SrRuO}_{3}(\mathrm{SRO}) / \mathrm{BaTiO}_{3}$ (BTO) bilayer on a $\mathrm{SrTiO}_{3}$ substrate. A dislocation is visible in the upper BTO layer. In the inset, the atomically-resolved HAADF images at both the sides of the domain wall are shown. (b) Rotation map highlighting the dislocation in the upper BTO layer. (c) Fourier filtering of the DPC signal to separate low and medium frequencies contributions. Applying a band-pass filter with a frequency range of $0.69-8.8 \mathrm{~nm}^{-1}$ (d) we retrieve the atomic electric field (shown for the region marked by the yellow box in (a)), while applying a low pass filter that selects the frequency lower than $0.69 \mathrm{~nm}^{-1}$ the mesoscopic polarization is obtained.

A similar result was previously obtained for the $\mathrm{Bi}_{0.8} \mathrm{Ca}_{0.2} \mathrm{FeO}_{3}$ system, in which spontaneously generated Cadopant fluctuations occurring in a layered fashion induce a complex polarization pattern with alternating polar (at the Ca-poor areas) and non-polar (Ca-rich areas) regions [56]. In Figure 9(a) a scheme of the polar pattern is shown, together with the HAADF and ABF images. The local change in the dopant composition is not effortlessly visible from the images, but it is linked to the alteration of the structural distortions responsible of the displacive ferroelectricity (see Ref [56]. for more details). Nonetheless, the layering of the dopant atoms generates a giant polarization gradient that is as large as $\sim 70 \mu \mathrm{C} \mathrm{cm}{ }^{-2}$ across only $3 \mathrm{~nm}$. Interestingly, the DPC-STEM signal highlights asymmetries in the electric field components (Figure 9(b)). The asymmetries are clearly due to the superimposition of the much localized atomic electric field and the slowly varying polarization. Indeed, the polarization variation occurs on a length scale significantly larger with respect to the size of the aberration-corrected STEM probe, inducing a small beam deflection that can be approximated as an additive term just superimposed to the atomic field. 

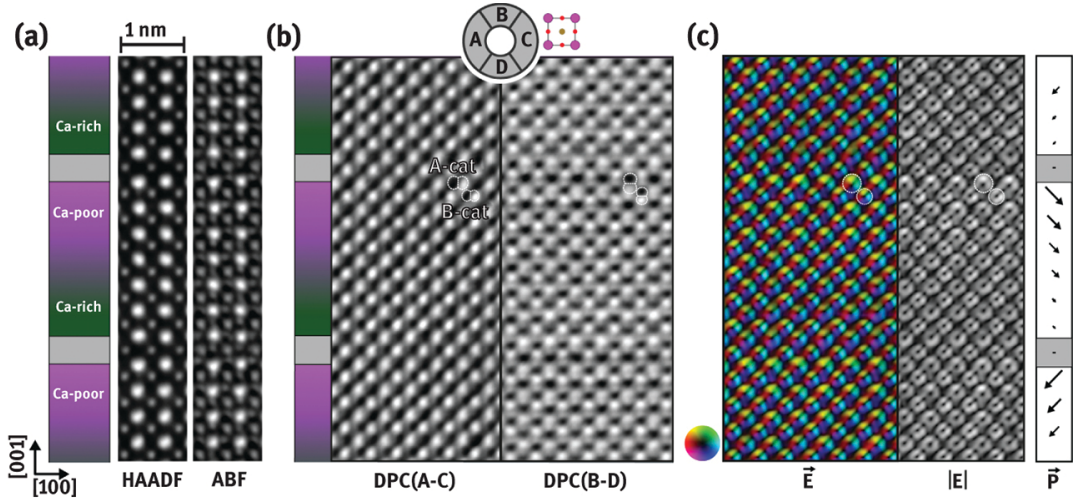

Figure 9: (a) Scheme of the Ca-dopant layering in a $\mathrm{Bi}_{0.8} \mathrm{Ca}_{0.2} \mathrm{FeO}_{3}$ thin film, together with the HAADF and ABF signals. (b) DPC-STEM signals and (c) vector and amplitude plot of the projected electric field. Reproduced from [56].

\subsubsection{Magnetic ordering in multiferroics}

Electron holography has been widely used to study the magnetic domain configuration in ferromagnetic and multiferroic specimens, including thin films [75-79], nanowires [80-83] and NPs [84-86]. Here, we report as an example the application of electron holography to multiferroic $\mathrm{Ni}_{2} \mathrm{MnGa}$ thin films and nanodisks.

$\mathrm{Ni}_{2} \mathrm{MnGa}$ is a multiferroic ferromagnetic shape memory alloy in which a spontaneous deformation as high as $6-10 \%$ can be induced by the application of a magnetic field. The ferroic properties of FMSAs arise as a consequence of coexistent ferromagnetic and ferroelastic (martensitic) phase transformations, and they give rise to a very peculiar nanostructure composed by twinned domains (usually called twin variants). The ferroelastic martensitic transformation results in martensite variants separated by mobile twin boundaries, which can be exploited to generate giant strains in FMSAs thin films and nanostructures. Figure 10(a) displays an electron hologram of a region showing twin variants oriented at $90^{\circ}$ with respect to the $\mathrm{MgO}(001)$ substrate. The mean inner potential and the magnetic phase contributions can be separated acquiring the hologram before and after turning upside-down the specimen. In Figure 10(b) and 10(c) the electrostatic and the magnetic phase shift are respectively shown. By calculating the gradient of the magnetic phase shift we can retrieve the magnetic induction components using eq. (12). The magnetic induction color map is shown in Figure 10(d) and the phase contours of the 6x amplified phase are presented in Figure 10(d). Electron holography allows thus for reconstructing the magnetic domain configuration in the twinned martensite and to map the magnetic induction flux lines.

(a)

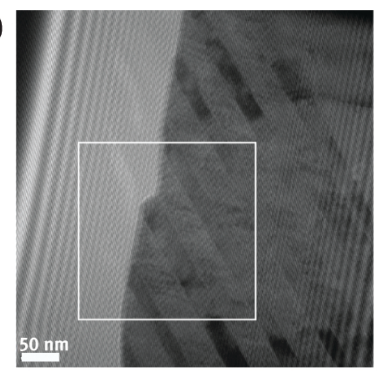

(c)

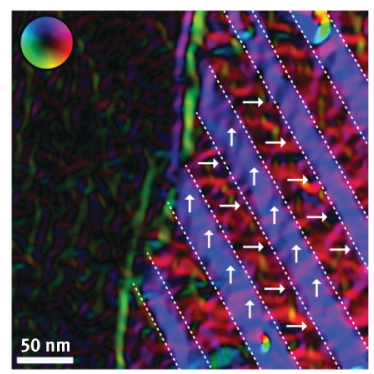

(b)

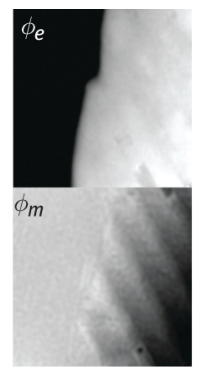

(d)

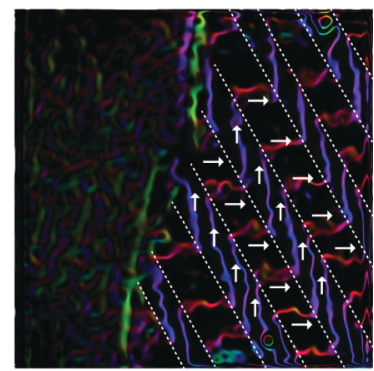

Figure 10: (a) $300 \mathrm{kV}$ electron hologram acquired using a $200 \mathrm{~V}$ biprism bias (fringe spacing: $1.4 \mathrm{~nm}$, fringe contrast: $20 \%$ ) on a $\mathrm{Ni}_{2} \mathrm{MnGa}$ thin film in a region showing $90^{\circ}$ twin variants. (b) Reconstructed electrostatic and magnetic phase shifts from the region marked by a white dashed box in (a). (c) Magnetic induction vector map and (d) magnetic induction flux lines of the 6x amplified phase. Adapted from [77]. 


\section{Electron nanodiffraction}

Compared to the above discussed phase-contrast methods, a completely different approach to extract local information of ferroic materials is based on electron diffraction. The most common method to access local diffraction information in TEM is selected-area electron diffraction (SAED). However, should diffraction information be required for sample areas in the nanometer range, SAED is unsuitable. In contrast, so-called electron nanodiffraction uses a small electron probe to record electron diffraction patterns. Typically the electron probes for electron nanodiffraction have diameters ranging from a few tens of nanometers to less than $1 \mathrm{~nm}$ depending on the selected objective and condenser lens settings. Thus, several nanodiffraction techniques exist that exploit different convergence angles [87-89]. Nanoarea electron diffraction (NAED or NED) uses the objective prefield lens to form a small (nearly) parallel probe on the specimen [90, 91]. By inserting a small condenser aperture, the beam size may be as small as a few tens of nanometers. In this mode, the diffraction patterns consist of sharp diffraction spots (comparable to the ones obtained by a parallel beam in a TEM). Instead, in nanobeam electron diffraction (NBED or NBD) a focused probe is formed by placing the electron beam crossover after the second condenser lens far away from the front focal plane of the OL, while keeping the mini-condenser lens weakly excited $[92,93]$. This gives a demagnified electron source image on the sample which can produce diffraction patterns from sub-nanometer regions. However, the diffraction patterns consist of small discs and, thus, NBED is only suitable for probing local structures where the absence of sharp diffraction spots is not critical, such as phase identification, orientation mapping and local strain measurements [88]. Schematic diagrams illustrating the electron paths in NAED and NBED modi are shown in Figure 11. By varying the excitation of the condenser lenses and apertures, the semi-convergence angle of the probe can be gradually changed between (nearly) parallel and convergent illumination. A gradual decrease of the convergence angle of the electron probe in NBED mode results in the NAED mode as a border case of vanishing convergence angle of the NBED mode. For each technique, the resulting electron probe and diffraction pattern from a $\mathrm{PbZr}_{0.2} \mathrm{Ti}_{0.8} \mathrm{O}_{3}$ thin film acquired using a probe aberration-corrected (scanning) transmission electron microscope, with a more complex condenser lens system than the one illustrated in Figure 11, are given as insets.

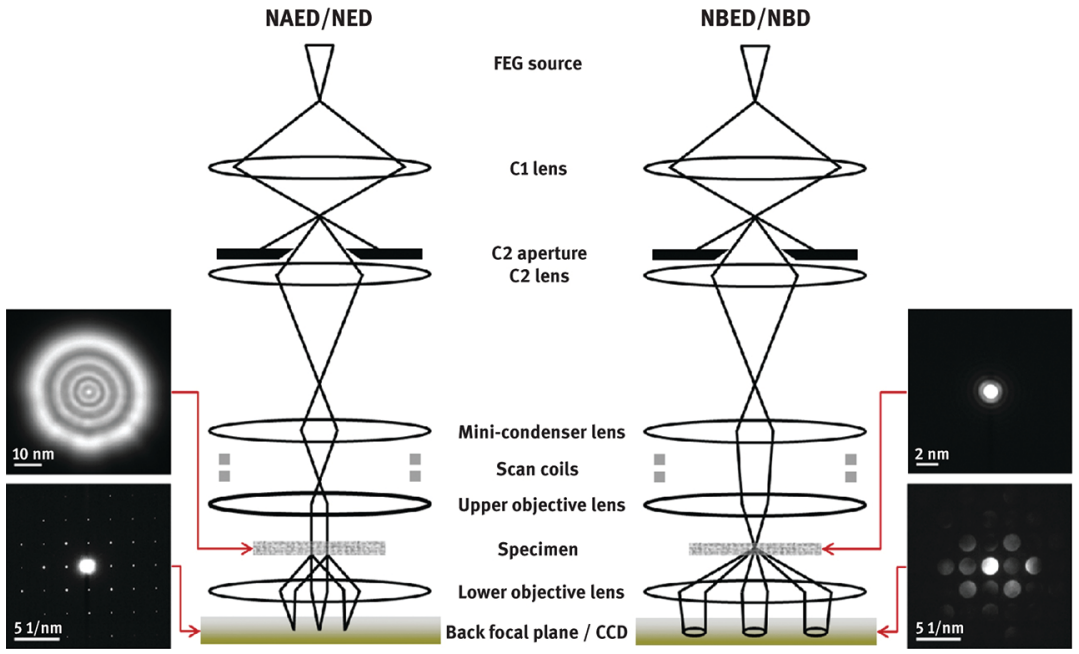

Figure 11: Ray diagrams showing the electron paths in NAED and NBED modes. For each technique, the resulting electron probe and diffraction pattern from a $\mathrm{PbZr}_{0.2} \mathrm{Ti}_{0.8} \mathrm{O}_{3}$ thin film are shown as insets. Left: the experimental NAED probe (obtained with a semi-convergence angle $<0.5 \mathrm{mrad}$ ) has a diameter of $\sim 50 \mathrm{~nm}$ and was used to record the diffraction pattern with lattice reflections showing rather sharp diffraction spots. Right: the full-width-at-half-maximum of the NBED probe (obtained with a semi-convergence angle $\sim 1.5 \mathrm{mrad}$ ) is $0.6 \mathrm{~nm}$ and was used to record the diffraction pattern consisting of small disks where the disk radius reflects the convergence angle of the electron probe. The ray diagrams are adapted from [87] and [91].

Additionally, electron nanodiffraction patterns can be obtained in conjunction with STEM imaging using an annular dark-field detector with a low camera-length setting. This operation mode is known as scanning electron nanodiffraction (SEND) [88]. In its simplest form, SEND can be carried out by positioning the electron probe, based on the STEM image, at a selected area of interest and recording diffraction patterns of this area. Alternatively, SEND patterns can also be automatically acquired from a predefined list of probe positions by scanning along a line or across an area. Indeed, conceptually this approach is identical to the above-mentioned 4D-STEM, except for the diffraction-specific electron probe and the information extracted from the diffraction patterns. When SEND patterns are acquired across an area, they can be used to form an image. Thus, bright- 
and dark-field STEM images can be obtained from SEND by integrating the diffraction intensities of the direct beam and selected diffraction beams, respectively. In this manner, SEND works like a STEM. But beyond the simple integrated intensities, other information can be extracted off-line to form images. For instance, the diffraction patterns can be indexed and analyzed to achieve phase and crystal orientation mapping. Also, full 2D strain maps can be extracted by accurately measuring the position of the Bragg diffracted beams from each image pixel [94-99]. However, the non-uniform contrast present in the large diffraction discs (due to variations of thickness and bending across the specimen field of view) can result in an incorrect determination of their position and deliver results that are incorrect. One way to remove the systematic errors arising from the noneven intensity in the spots is to precess the electron beam around each sampling point. This approach known as nanobeam precession electron diffraction (NPED) leads to uniform illumination in the diffracted beams and allows the position of each spot to be more accurately determined [100]. Additionally, a higher convergence angle can be used to form a smaller probe for improved spatial resolution.

Both nanobeam modes, NBED and NPED, have been extensively used in the semiconductor industry to measure local strains in nanodevices, identify crystal defects and determine crystal structures. However, there are very few publications on electron nanodiffraction studies of ferroelectric and multiferroic materials [101]. Yet, the experiments reported to date suggest important possibilities for the detailed structural characterization of multiferroics in the ultrathin regime or inserted in multilayers or superlattice architectures.

\section{Strain mapping techniques}

Driven by the need of developing new semiconductor devices substantial progress has been made in the last decade in the advancement of transmission electron microscopy based techniques for strain mapping at high spatial resolution. Such developments are also very valuable to the research field of multiferroic magnetoelectric materials as their ground state can be tuned by epitaxial strain to adopt very different multiferroic phases exhibiting a number of fascinating phenomena. Thus, the exploration of epitaxial strain due to lattice mismatch is critical for the design and growth of epitaxial multiferroic thin films for next-generation power-efficient oxide electronics. Currently, the TEM techniques that provide the best spatial resolution (below $5 \mathrm{~nm}$ ) and quantitative strain maps are: nanobeam electron diffraction (NBED) and its more advanced version NPED in scanning mode, dark-field electron holography (DFEH), high-resolution transmission electron microscopy (HRTEM or HREM) and high-angle annular dark-field scanning transmission electron microscopy (HAADF-STEM) [88, 99, 102].

\subsection{Strain mapping from SEND techniques}

The basic principles of NBED and NPED were introduced in the previous section. The main advantage of NPED over NBED is that a higher convergence angle can be used to form a smaller probe, thus, improving the lateral spatial resolution. Additionally, NPED is very insensitive to thickness variations in the specimen leading to more robust results. Hence, from these two reciprocal space techniques, NPED provides the best combination of spatial resolution $(2-5 \mathrm{~nm})$ and precision $(0.02-0.05 \%)$ [99]. However, both NBED and NPED require additional hardware control (an additional condenser lens and precession coils, respectively) and the data sets can easily contain thousands of diffraction patterns making off-line data processing lengthy and heavy.

\subsection{Strain mapping from Dark Field Electron Holography}

DFEH is a variant of off-axis electron holography introduced earlier in this review. In DFEH, an electron beam diffracted by a set of lattice planes is first selected using an objective aperture. Second, a biprism placed below the specimen is used to interfere the electron beams that have been diffracted by both the strained region of interest and a reference region, usually taken in the substrate. Thus, an interference pattern (or dark-field hologram) is obtained and used to calculate the relative phase of the diffracted beam between the two regions $[103,104]$. The phase and exact location of a diffracted beam depend on the crystal spacing. The phase of a diffracted beam has an additional contribution given by $\varphi(r)=2 \pi g \cdot u(r)$, where $\mathbf{g}$ is the scattering vector and $\mathbf{u}(\mathbf{r})$ the displacement field as a function of the position $\mathbf{r}$ [104]. Since the transmitted beam has $\mathbf{g}=\mathbf{0}$, it does not carry any information about the phase, and it is needed to select a diffracted beam for this type of holography. Hence, in order to do DFEH one needs to form a dark-field image [4] with a selected diffraction spot using an objective aperture, place the biprism between the strained and the reference area, and form an interference pattern within that dark-field image of the strained and the reference area. From this interference pattern, the 
strain map in the direction of the selected diffraction spot can be deduced. By repeating the same procedure for at least another linearly independent diffraction spot the full-field strains can be mapped.

For the measurement of strain, the specimens are required to be thin $(<100 \mathrm{~nm})$ and flat in order to ensure uniform diffraction conditions over the two regions. Any slight bending of the thin specimen is particularly prejudicial to obtain a homogeneous dark-field contrast [105]. Additionally, DFEH is often considered practically difficult because the intensity of dark-field holograms is low, and highly stable microscopes are needed for long acquisition times [106]. Nevertheless, DFEH is particularly attractive since strain maps with a large field of view (up to $1 \mu \mathrm{m}$ ) and high sensitivities (as low as 0.02-0.05\%) can be obtained [99, 107, 108]. Moreover, the DFEH technique excels in its speed of both data acquisition and processing of strain maps. As such, this technique has been extensively used for measuring strain in semiconductor devices and thin films [103, 105, 108]. But then again, there has been very limited research on quantitative measurements of displacement and strain fields by DFEH from multiferroic/ferroelectric films. Perhaps the most relevant example is the work by Denneulin et al. [109] on the deformation fields in epitaxial $\mathrm{Pb}\left(\mathrm{Zr}_{0.2} \mathrm{Ti}_{0.8}\right) \mathrm{O}_{3}$ films containing $90^{\circ}$ ferroelastic domains; see Figure 12. For the conditions used in their paper, they were able to measure the deformation gradients in $\mathrm{Pb}\left(\mathrm{Zr}_{0.2} \mathrm{Ti}_{0.8}\right) \mathrm{O}_{3}$ with a sensitivity of $0.1 \%$ and a spatial resolution of $6 \mathrm{~nm}$ over fields of view up to $500 \mathrm{~nm}$. Thus, we anticipate that the ability of DFEH to measure strain over very large areas shall become very valuable when it comes to quantitatively measure strain in future nanoscale multiferroic devices and sensors.
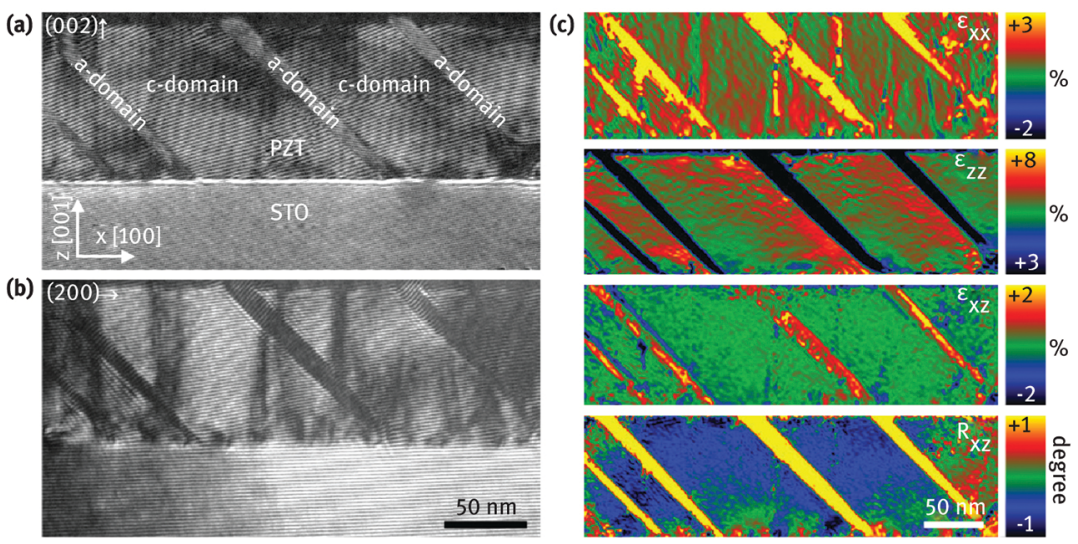

Figure 12: Local deformation gradients in an epitaxial $\mathrm{Pb}\left(\mathrm{Zr}_{0.2} \mathrm{Ti}_{0.8}\right) \mathrm{O}_{3}$ film by DFEH. (a) (002) and (b) (200) dark-field electron holograms of a $100 \mathrm{~nm}$ thick film containing a- and c-domains. (c) From top to bottom: $\varepsilon_{\mathrm{xx}}$ in-plane, $\varepsilon_{\mathrm{zz}}$ out-ofplane, $\varepsilon_{\mathrm{xz}}$ shear deformation maps and $\mathrm{R}_{\mathrm{xz}}$ rigid-body rotation map. Adapted from [109].

\subsection{Strain mapping from high-resolution images}

HRTEM and HAADF-STEM allow imaging the structure of materials at the atomic scale. Additionally, with the development of aberration correctors, sub-Ångström resolution images can now be routinely obtained permitting the determination of atomic column positions with picometer precision. Then, by measuring the lattice distortions and/or local atomic displacements in the images quantitative strain maps can be extracted, provided a known reference area is present in the image. These techniques provide the best spatial resolution but at the expense of a limited field of view.

The key difference between HRTEM and HAADF-STEM lies in their modes of image formation. In TEM, an electron plane wave illuminates the specimen and the OL forms a magnified image, which is projected to the image plane. In the STEM mode, a finely focused electron probe is scanned across the specimen and the transmitted and/or scattered electrons from a localized volume of the specimen are collected on post-specimen (extended discs and/or annular) detectors as a function of raster position. TEM provides almost perfect coherent imaging whereas the STEM instrument can be configured for almost perfect incoherent imaging [110]. Hence, among the two techniques, there has been increased interest in measuring strain from HAADF-STEM images because peak intensities are registered at the atomic positions and are dependent on the atomic number (Z-contrast) over a large range of sample thicknesses, so that the images are directly interpretable. On the contrary, in HRTEM changes of specimen thickness and defocus can cause atomic columns to reverse contrast introducing artifacts in the deformation maps. Thus, atomically centered contrast in HRTEM is only accessible when the sample is at an optimal defocus and very thin. Moreover, on aberration-corrected microscopes, the aberrations (defocus, spherical aberrations) need to be balanced to optimize the phase-contrast that dominates the image contrast in HRTEM. Here, specimen preparation remains a practical challenge, especially when large fields of view are desired. 
However, a major issue of using STEM imaging for strain measurements is the noise present in the images. As image intensities are acquired pixel by pixel, the recorded image is affected by relative movements of the electron probe and sample during acquisition, as well as systematic and random noises in the probe scan. Random scan noise is introduced due to sudden beam position changes (fly-back distortion) in conventional line-by-line raster scans, while systematic noise might arise from sample drift, environmental interferences, deviations from the saw-tooth deflecting voltage applied to the scan coils, or any systematic scan noise introduced during the scan fly-back time [89]. These are serious issues which can heavily degrade the quality of the STEM images and worsen the strain mapping precision. Hence, a stable environment (room conditions) and a stable microscope platform are essential for quantitative STEM measurements. For remaining instabilities, many approaches have been proposed to tackle the unwanted noise affecting STEM images. These can be grouped into those using reference data or those using frame-averaging [111].

Reference approaches assume that distortions remain constant between images recorded at different times $[112,113]$ or within whole scan-lines of an image [114]. However, as actual environmental distortions cannot be assumed to be reproducible, such methods often preserve scan-distortion artifacts in their corrected data [113115]. This is illustrated in the example of Figure 13, where it is shown that residual scan-distortion artifacts are preserved in the corrected data.
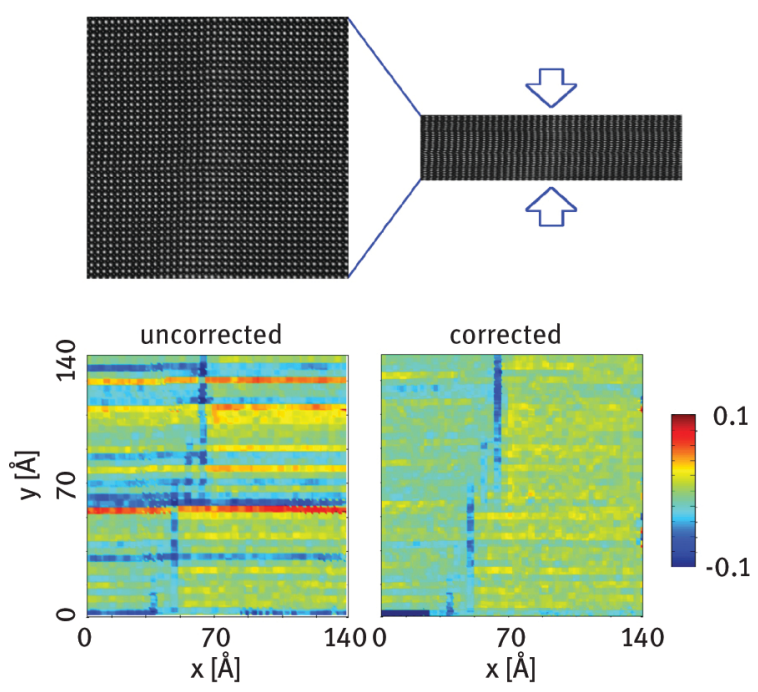

Figure 13: Effect of random scanning errors on the strain analysis of $109^{\circ}$ domain walls in $\mathrm{BiFeO}_{3}$. Top: HAADF-STEM image of a stepped domain wall running vertically across the whole field of view. If the image is vertically squashed, the atomic lattice no longer appears straight. Bottom: the unit-cell-wise shear strain $\varepsilon_{x z}$ is depicted before and after removing the scanning errors, which are visible as characteristic horizontal streaks. Note that although most of the scan-distortion artifacts are removed, some are preserved in the corrected data. They are seen in blue and yellow horizontal strikes at both sides of the stepped domain wall. Adapted from [115].

Multi-frame acquisition approaches combined with post-processing have demonstrated significant improvements in precision and show an increased signal-to-noise ratio. These approaches include simple rigidalignment [116], more advanced rigid-plus-affine registration of revolving image series [117], unconstrained non-rigid registration [118], and non-rigid registration using the "row-locking" technique [119]. Remarkably, sub-pm precision in the determination of atomic positions has been achieved by non-rigid registration and averaging of series of 20-30 short-exposure images [118, 119]. The resulting distortion-corrected images can be subsequently analyzed in order to obtain distortion and/or strain maps with high precision.

An example of a noisy single HAADF-STEM image and the analogous distortion-corrected image obtained from averaging several images non-rigidly is given in Figure 14 and Figure 15. In Figure 14, a single HAADFSTEM image acquired with $20 \mu \mathrm{s}$ dwell time of a $\mathrm{BiFeO}_{3}$ thin film on a $\mathrm{La}_{0.7} \mathrm{Sr}_{0.3} \mathrm{MnO}_{3}$ layer reveals the presence of two edge dislocations [79]. Here, the enlarged view (inset) of the area highlighted with a square clearly shows the presence of errors in the beam position along the fast scan axis (also known as "flags"). The errors in the beam position along the slow scan ("skips") lead to an extension or truncation of the image and are perceptible as horizontal stripes in the $\varepsilon_{z z}$ and $\varepsilon_{x z}$ strain and $R_{x z}$ rotation maps. The analogous image obtained by non-rigid registration of 20 HAADF-STEM images acquired with $1 \mu \mathrm{s}$ dwell time is shown in Figure 15. Both the enlarged view and the corresponding strain and rotation maps demonstrate that the flags and skips have been corrected resulting in a significant improvement in the strain precision. 

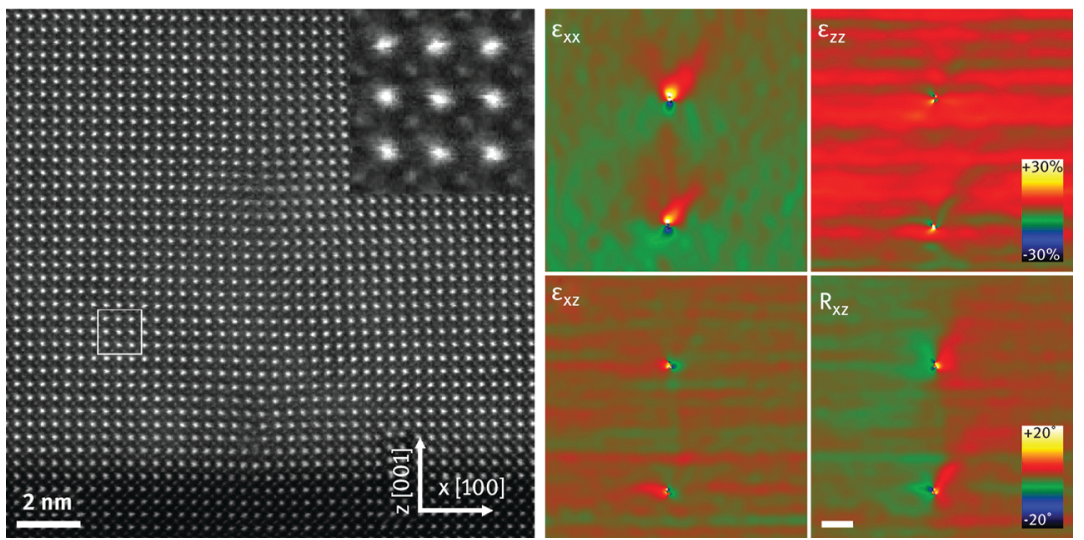

Figure 14: Single HAADF-STEM image acquired with $20 \mu$ s dwell time of a $\mathrm{BiFeO}_{3}$ thin film on a $\mathrm{La}_{0.7} \mathrm{Sr}_{0.3} \mathrm{MnO}_{3}$ layer revealing the presence of two edge dislocations at a distance of $8 \mathrm{~nm}$. The inset is an enlarged view of the area highlighted with a square showing the errors in the beam position along the fast scan axis (flags). The errors in the beam position along the slow scan (skips) lead to an extension or truncation of the image and are perceptible as horizontal stripes in the $\varepsilon_{\mathrm{zz}}$ and $\varepsilon_{\mathrm{xz}}$ strain and $\mathrm{R}_{\mathrm{xz}}$ rotation maps obtained by GPA. These fluctuations are absent in the $\varepsilon_{\mathrm{xx}}$ strain map because the scanning direction is parallel to the principal axis of the strain. The color bars give the change in strain and the rigid rotation angle in percent and degrees, respectively. The scale bar is $2 \mathrm{~nm}$.
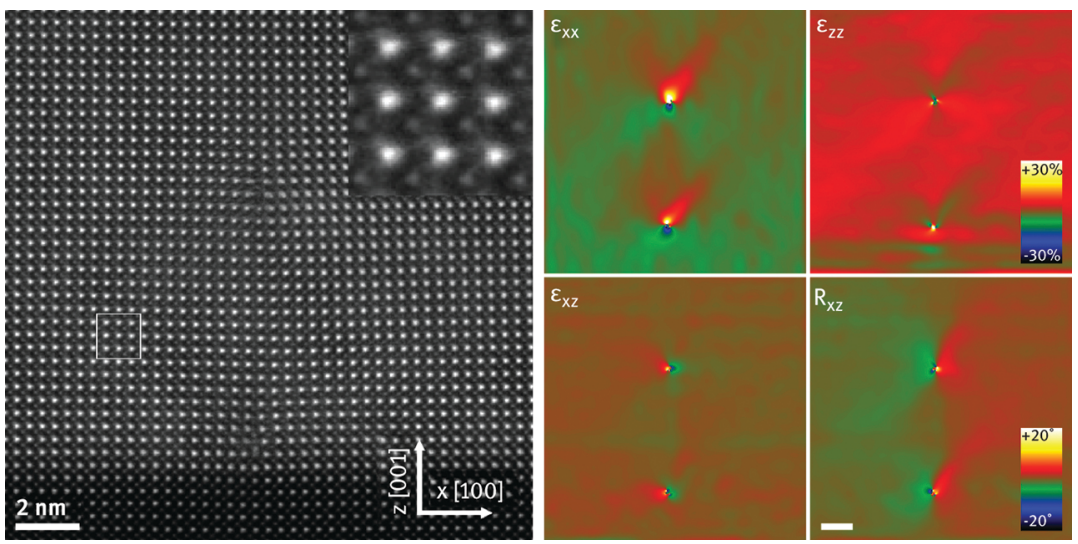

Figure 15: Non-rigid registration of 20 HAADF-STEM images acquired with $1 \mu$ s dwell time of the same area shown in Figure 14, and corresponding strain and rotation maps obtained by GPA. The inset is an enlarged view of the area highlighted with a square. Note that the flags and skips have been corrected. The color bars give the change in strain and the rigid rotation angle in percent and degrees, respectively. The scale bar is $2 \mathrm{~nm}$.

Several methodologies have been described in the literature to obtain strain maps from HRTEM and HAADFSTEM images, either in Fourier space (GPA) or in real space (Peak Finding). They provide the best spatial resolution $(1-5 \mathrm{~nm})$ but a relatively low precision $(0.2-0.4 \%)$ as compared to the previously described deformation mapping techniques [99].

Geometric Phase Analysis (GPA) works in the Fourier space and consists of measuring the local displacements of atomic planes by calculating the local Fourier components of the lattice fringes in a HRTEM or HAADF-STEM image [120]. This is done by centering a small aperture on a Bragg spot in the Fourier transform of the lattice image and performing an inverse Fourier transform. The phase component of the resulting complex image contains information about the local lattice displacements and the 2D displacement field can be derived by applying the method to two or more non-collinear Fourier components. Subsequently, the local strain components can be obtained by computing the derivative of the displacement field [121,122]. The spatial resolution of the GPA technique is determined by the size of the mask used to extract the Bragg spot.

Peak-Finding methods work in real-space and take advantage of all information recorded in an atomic resolution HRTEM or HAADF-STEM image. They are based on locating all the intensity maxima registered at the atomic columns and superimposing a $2 \mathrm{D}$ reference lattice extrapolated from a distortion-free region of the material in order to calculate the local discrete displacement field at each node. Then, by derivation of the calculated displacement field, the strain field is obtained [114, 123]. This methodology works very well for the analysis of pseudomorphic heterostructures but the automatic image girding usually fails at defective regions, such as dislocations cores. At these positions human intervention is typically needed to correctly construct the lattice. An alternative real space algorithm based on the detection of pairs of intensity maxima was proposed by Galindo et al. [124]. The algorithm, known as Peak Pairs Analysis (PPA) [125], works on a filtered image by 
locating pairs of peaks along two predefined basis vectors. From the deformation of each pair across the image, the displacement field can be reconstructed and used to calculate strain maps. PPA exhibits a good behavior at heavily distorted areas, for instance at interfaces of plastically relaxed systems, without user's intervention. However, PPA fails when lattice peaks are not clearly detected due to static tilts of atomic columns near specific dislocations or grain boundaries. In these cases, GPA is recommended [124].

The TEM techniques introduced in this section are very valuable to the research field of multiferroics as the nanoscale strain gradients are known to influence different functional properties of the films [79, 126-130]. Thus, if properly exploited, the epitaxial strain can be used as an important factor to tailor the macroscopic response toward desired properties, such as ferroic transition temperatures, magnetic order and ferroelectric polarization.

\section{Ferroelectric polarization mapping from high-resolution images}

Aside from local strain measurements, atomic-resolution micrographs also allow for assessing the local symmetry of individual unit cells and thus for indirectly measuring the local polarization of the sample (see DOI: 10.1515/PSR.2019.0015). The ferroelectric polarization can be determined from the atomic displacements measured in atomic resolution images. Earlier reports on polarization mapping mostly used HRTEM techniques such as negative spherical aberration (Cs) condition [131] or exit wave reconstruction of focal series [74]. However, in the last few years HAADF-STEM has emerged as the preferred technique for mapping atomic polar displacements because the samples can be thicker, which helps to preserve the native domain structure, and the interpretation of the image contrast is straightforward and reliable [56, 79, 115, 132-139]. Mapping of atomic polar displacements from HAADF-STEM images was successfully applied to reveal various domain wall configurations [115, 132-135, 140], flux-closure domain patterns [136], ferroelectric vortex domains [137, 138], polarization gradients [56,141], as well as interface and surface reconstructions in ferroelectric thin films [142]; see Figure 16. In these examples, the ferroelectric films present the pseudocubic perovskite-type structure with general formula $\mathrm{ABO}_{3}$, where $\mathrm{A}$ and $\mathrm{B}$ are cations of different size and $\mathrm{O}$ is the anion bonding to both. In the perovskite structure, the larger A cations lie in the center of 12 oxygen ions in dodecahedral coordination, and the smaller $\mathrm{B}$ cations are octahedrally coordinated. The polar displacement resulting from the off-centering of the cations with respect to the oxygen octahedra induces spontaneous polarization. However, in the HAADF-STEM images, the light elements such as oxygen are usually undetectable when the compounds contain relatively heavier atoms; then polarization maps are effectively calculated from the relative displacements of the two cation sublattices. As the B cations are displaced along with the oxygen octahedra, the displacement vector can be determined by measuring the polar displacement of the $\mathrm{B}$ position in the image plane from the center of its four $\mathrm{A}$ neighbors. Thus, the displacement vector as the manifestation of the ferroelectric polarization in $\mathrm{ABO}_{3}$ points toward the center of the negative oxygen ions and can be used to determine the polarization vector. In the polarization maps derived from HAADF-STEM images, the polarization vectors are typically plotted opposite to the polar displacement of the $B$ cations. An example of a polarization map, superimposed on a HAADF-STEM image of a $\mathrm{Bi}_{0.8} \mathrm{Ca}_{0.2} \mathrm{FeO}_{3}$ thin film, calculated from the displacement of the Fe cation with respect to the CoM of the Bi cell, is shown in Figure 17(a). 

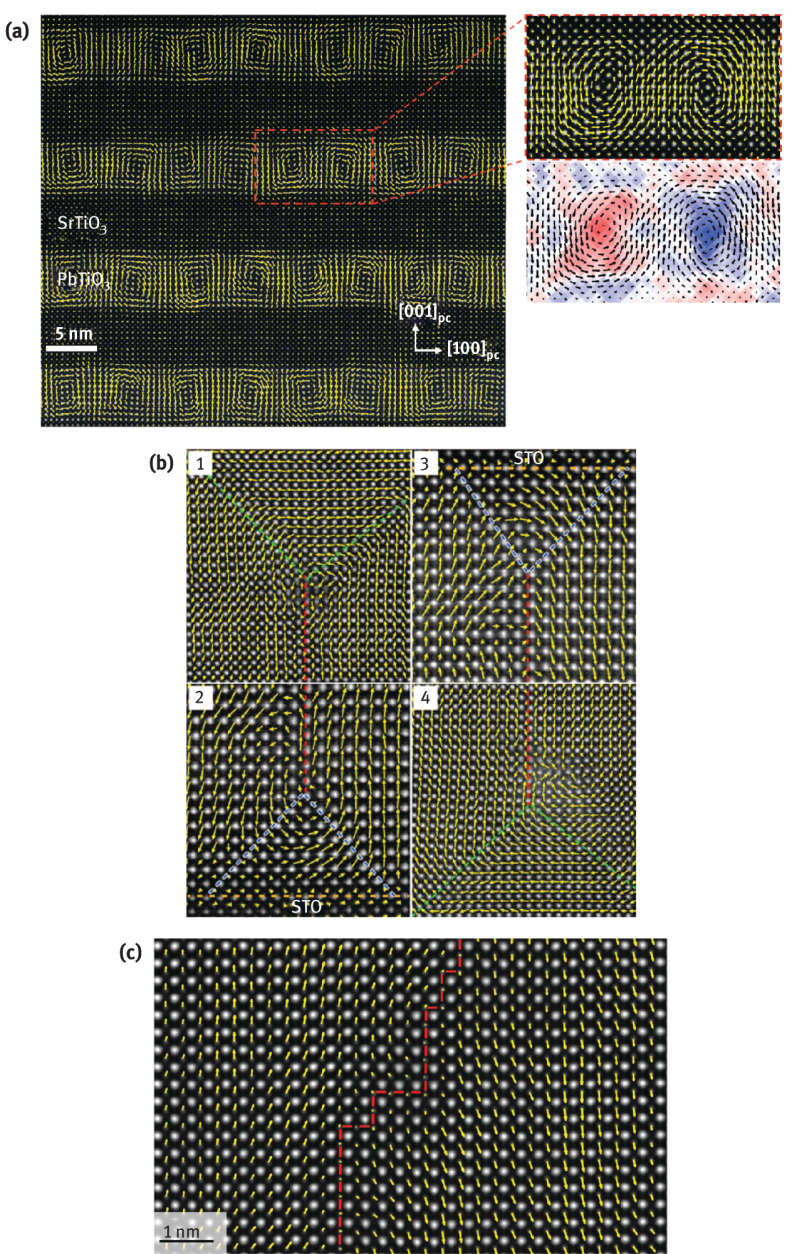

Figure 16: Mapping of atomic polar displacements from HAADF-STEM images. (a) Ferroelectric vortex domains in a $\left(\mathrm{SrTiO}_{3}\right)_{10} /\left(\mathrm{PbTiO}_{3}\right)_{10}$ superlattice [138]. The curl of the polar displacement $(\nabla \times P)_{[010]}$ is plotted for a vortex-antivortex pair with a red/blue color scale; clockwise (negative) is blue and anticlockwise (positive) is red. (b) Flux-closure domains in strained $\mathrm{PbTiO}_{3}$ films [136]. (c) $180^{\circ}$ domain wall in a $\mathrm{PbZr}_{0.2} \mathrm{Ti}_{0.8} \mathrm{O}_{3}$ film displaying a mixed Ising-Néel type character [135]. 

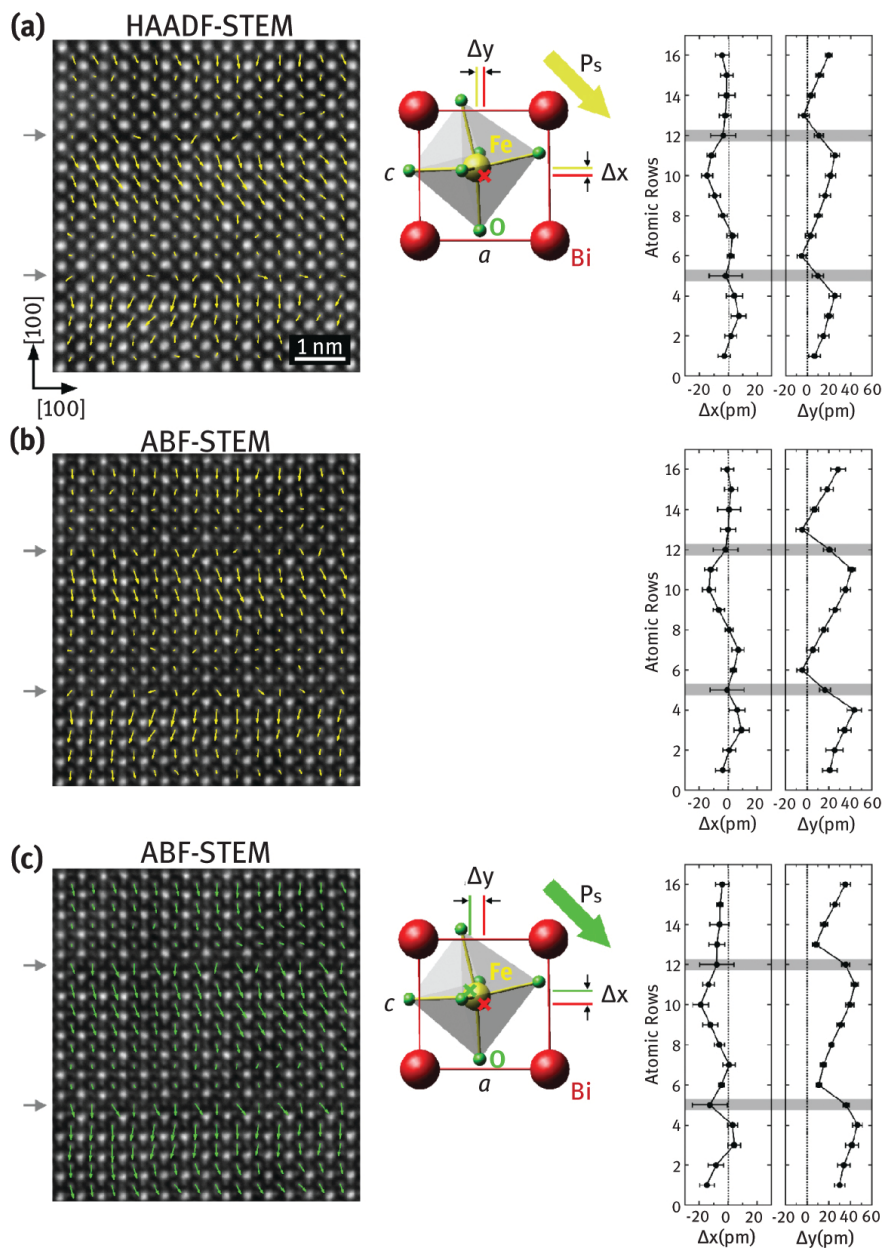

Figure 17: Periodic giant polarization gradients in $\mathrm{Bi}_{0.8} \mathrm{Ca}_{0.2} \mathrm{FeO}_{3}$ thin film. Polarization maps superimposed to (a) an averaged HAADF-STEM image and (b) the simultaneously recorded inverted ABF-STEM image calculated from the displacement of the Fe cation with respect to the CoM of the Bi cell (marked with a red cross in the model). (c) Polarization map superimposed to the inverted ABF-STEM image calculated from the relative displacements of the CoM of the oxygen octahedron and the CoM of the Bi cell (marked with green and red crosses in the model, respectively). As indicated in the models, the polarization vectors are plotted opposite to the polar displacements of the $\mathrm{B}$ cations and $\mathrm{O}$ anions. The corresponding profiles along the [001] direction of the in-plane $(\Delta x)$ and out-of-plane $(\Delta y)$ components of the atomic displacements are shown at the right side. The gray rectangles indicate the position of two oxygen deficient layers. Adapted from [56].

A more straightforward way of obtaining polarization maps can be attained, in principle, by using annular bright-field (ABF) STEM imaging as it allows for simultaneously visualizing both heavy and light atomic columns in a single image [143-148]. This imaging mode uses an annular detector spanning an angular range within the direct scattering cone, which is defined by the probe-forming aperture of the focused electron beam $[143,147]$. In ABF-STEM the image contrast is mostly dominated by elastically scattered electrons and the images show, for suitable defocus values, a negative absorptive-type signal at all (light and heavy) column locations over a large range of thicknesses [147, 149]. Therefore, this imaging mode has turned out to become an important imaging mode for the quantitative structure determination of systems composed of a mixture of light and heavy atoms, such as oxides and perovskites [56, 139, 150]. However, unlike HAADF-STEM imaging, ABF-STEM images are highly sensitive to sample mistilt [147, 151-153]. Specifically, the misalignment of the sample results in significant column-type dependent shifts, especially for thicker specimens. Recent work on the accuracy of ABF-STEM imaging revealed that a small sample mistilt of about 6 mrad relative to the electron beam causes an artificial displacement of $11.9 \mathrm{pm}$ between the oxygen and cation columns for cubic $\mathrm{SrTiO}_{3}$ [152]. Artifacts of this magnitude can thus result in the depiction of a deformed unit cell preventing the reliable quantification of lateral atomic displacements based on ABF-STEM images. This is exemplified in Figure 17. By comparing the profiles in Figure 17(a and b), one can easily observe that the out-of-plane ( $\Delta y)$ relative displacements of the two cation sublattices are much larger for the ABF-STEM image $(\sim 47 \mathrm{pm})$ than for the HAADF-STEM image $(\sim 30 \mathrm{pm})$. Further, the profile in Figure 17(c), calculated from the relative displacements of the CoM of the oxygen octahedron and the CoM of the Bi cell, is shifted laterally away from the zero-displacement axis as a result of a small sample mistilt around the [100] axis. When using ABF-STEM imag- 
ing for polarization mapping it is therefore extremely important to align the sample's zone axis to the optical axis as accurately as possible, i. e. within a precision of tilt of $<1-2 \mathrm{mrad}$, which is typically the limit of the tilt accuracy of the TEM goniometer [151].

For a practical application of the above outlined experimental approach we discuss a non-trivial example, namely the multiferroic hexagonal manganites $\mathrm{h}-R \mathrm{MnO}_{3}(R=\mathrm{Sc}, \mathrm{Y}, \mathrm{Dy}-\mathrm{Lu})$, which belongs to a family of materials attracting much interest, particularly because of the observation of antiphase structural domains clamped to ferroelectric domain walls [154-160] (as discussed in DOI: 10.1515/PSR.2019.0014). The structural domains, when arranged in a particular phase relation forming a clover-leaf pattern, result in the appearance of structural vortices, which in turn induce magnetic vortices [161]. Additionally, it was shown that in hexagonal manganites ferroelectric domain walls can carry a magnetic moment [161-163].

The high-temperature paraelectric $R \mathrm{MnO}_{3}$ structure displays $P 6_{3} / m m c$ symmetry and consists of cornerlinked $\mathrm{MnO}_{5}$ trigonal bipyramid layers alternating along the $c$-axis with $R$ layers. At the symmetry-lowering phase transition, polarization emerges as a result of periodic tilts of the $\mathrm{MnO}_{5}$ bipyramids and displacements of the $R$ ions along the $c$ axis; see Figure 18. This periodic $\sqrt{3} \times \sqrt{3} \times 1$ lattice distortion, which is driven by the condensation of a zone boundary $K_{3}$ phonon, triples the crystallographic unit cell and is often referred to as the trimerization transition. Thus, the $R$ ions arrange in the form of sinusoidal waves and display either a $\uparrow \uparrow \downarrow$ pattern with positive $(+)$ polarization or a $\downarrow \downarrow \uparrow$ pattern with negative $(-)$ polarization. Each polarization pattern exhibits three possible permutations resulting in three distinct $\varphi$ structural domains $(\alpha, \beta$, and $\gamma)$. Therefore, the low-temperature ferroelectric phase, $P 6_{3} \mathrm{~cm}$, presents a total of six structural domains, labeled $\alpha^{+}, \beta^{-}, \gamma^{+}, \alpha^{-}$, $\beta^{+}, \gamma^{-}$. By driving the system through this structural transition, topological defects are created in the form of discrete vortices around which $\varphi$ changes monotonically in a clockwise or counterclockwise fashion. The core region is typically about $3 \mathrm{~nm}$ in diameter and exhibits nearly zero polarization [164, 165].
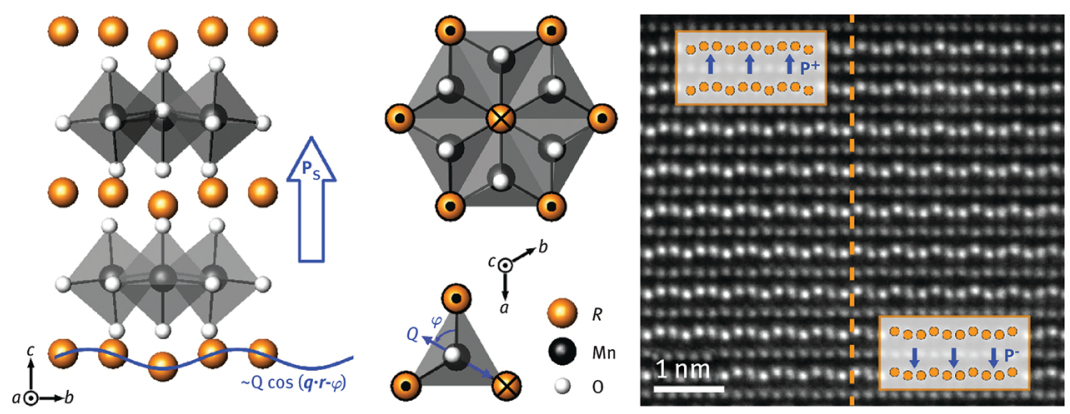

Figure 18: Ferroelectric order in hexagonal manganites. The side and top views of the $P 6_{3} \mathrm{~cm}$ unit cell show the lattice trimerization $\mathbf{Q}$ with tilt amplitude $Q$ (in this case away from the common center) and azimuthal tilt angle $\varphi$ of the $\mathrm{MnO}_{5}$ bipyramids, and the resulting sinusoidal corrugation pattern of the $R$ atoms. For clarity, the oxygen atomic displacements for one of the three bipyramids constituting the tilt are indicated. The HAADF-STEM image corresponds to a neutral domain wall in $\mathrm{YMnO}_{3}$ (marked by the broken line) separating two neighboring domains with opposite polarization. The insets show the $\mathrm{Y}$ displacements observed at each side of the domain wall.

The crystal-lattice trimerization can be fitted by using the model proposed by Holtz et al. [165]:

$$
u=Q \cos (\boldsymbol{q} \cdot \boldsymbol{r}-\varphi)
$$

where $\boldsymbol{q}$ is the wavevector of the $K_{3}$ mode, $r$ the position vector of the $R$ atom, $Q$ the amplitude of the sinusoidal wave and $\varphi$ its phase, with discrete values given by $\varphi=n \pi / 3(\mathrm{n}=0,1, \ldots, 5)$. The primary order parameter of the structural trimerization is hence given by $Q=(Q \cos \varphi, Q \sin \varphi)$. Noteworthy, the two parameters $Q$ and $\varphi$ are connected to remarkable physical quantities: $Q$ is related to the maximum displacement $(1.5 \times Q)$ between $R$ atoms, and $\varphi$ is associated with the six possible structural domains generated by the possible permutations of the $\uparrow \uparrow \downarrow$ and $\downarrow \downarrow \uparrow$ patterns.

From Landau theory, the polarization $P$ can be directly calculated from the values of $Q$ and $\varphi$ from the relation [165]:

$$
\mathbf{P} \sim Q^{3} \cos 3 \varphi
$$

Therefore, from the fitted values of $Q$ and $\varphi$, the polarization can be calculated. The paired charge density due to the material polarization can then be calculated using the equation:

$$
\rho_{P}=-\nabla \cdot \mathbf{P}
$$


and allows a direct visualization of the charged domain walls.

As an example, Figure 19 shows a HAADF-STEM image of $\mathrm{YMnO}_{3}$, where the displacement pattern of the $\mathrm{Y}$ atoms changes gradually across three $180^{\circ}$ domain walls. Thus, four distinct ferroelectric domains can be identified. Additionally, the charge density map reveals that, due to the non-straight nature of the domain walls, in some areas the polarization is not parallel to the wall, so that $\nabla \cdot \mathbf{P} \neq 0$. This example demonstrates that by carefully applying the above methodology critical structural information about complex multiferroics can be obtained, on length scales which are difficult to access by other methods.
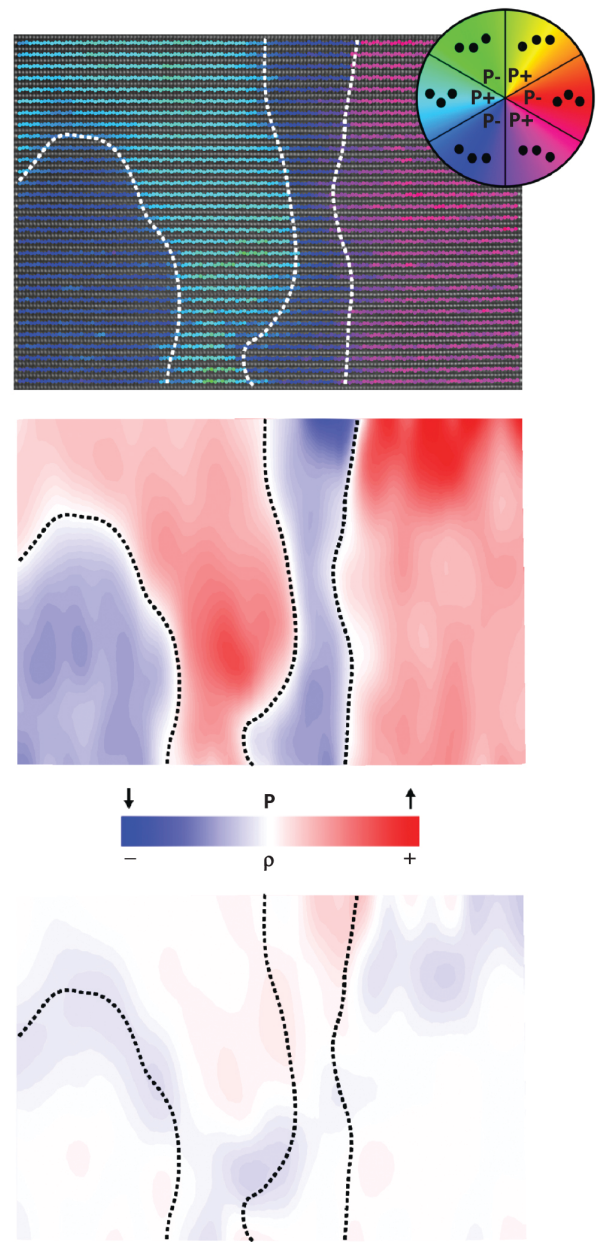

Figure 19: Charged domain walls in $\mathrm{YMnO}_{3}$. Top: HAADF-STEM image with a color overlay of the trimerization phase. The displacement pattern of the $\mathrm{Y}$ atoms changes gradually across the $180^{\circ}$ domain walls. Middle: plot of the polarization. Red indicates polarization up and blue polarization down, according to the color bar. Bottom: charge density map. Positively and negatively charged walls are indicated in red and blue, respectively.

\section{New possibilities of in situ measurements}

The success of in situ TEM measurements has been facilitated in recent years by the development of a number of technologies. Progress in Micro-Electro-Mechanical Systems (MEMS) has led to the development of dedicated TEM holders providing different stimuli (such as heating, electrical biasing, mechanical force, etc.) or a combination of multiple stimuli at once. The investigations can moreover be carried out in vacuum, gas or liquid environment. Additionally, the introduction of scanning/transmission electron microscopes with subÅngström resolution as well as fast and sensitive detection cameras capable of high speed (with more than 1000 frames per second) support the direct observation of dynamic phenomena in situ down to the atomic scale.

This section covers the latest developments in in situ TEM instrumentation which are of relevance to characterize magnetoelectric multiferroics. In particular, we describe here recent advances of in situ MEMS-based holders for heating and electrical biasing experiments and provide relevant literature on in situ observations of domain structure evolution and phase transitions. 


\subsection{Heating stages}

For many years the only available in situ heating stages for TEM were furnace type holders made from tantalum or Inconel, and designed to accommodate standard $3 \mathrm{~mm}$ disk specimens. These holders can reach $1200{ }^{\circ} \mathrm{C}$ (when using tantalum), but require the use of circulating water to cool down the specimen rod and to maintain the targeted temperature at the specimen. Hence, these traditional systems suffer from slow response times, (comparably) inaccurate temperatures and severe sample instability complicating atomic resolution imaging.

To address these issues, microfabricated heating stages with MEMS chips as disposable cartridges were developed $[166,167]$ and have become commercially available. The MEMS chips are typically made of silicon as the main body and present a window which is covered with a free-standing non-electron transparent membrane consisting of an electrical insulator material (usually silicon carbide or silicon nitride) [167-170]. The membrane is offered with either electron transparent windows made of amorphous silicon nitride or carbon (for nanostructures) or through-holes (for FIB lamella applications or 2D materials). The cartridges incorporate integrated circuitry which is used to pass an electrical current and, thus, to produce localized resistive heating directly on a small region of the chip of about $0.1 \mathrm{~mm}^{2}$. The Protochips systems [171] utilize ceramic heaters and each chip is calibrated by the supplier (using an optical pyrometer) and is provided with a chip-specific calibration file. Instead, the other systems $[172,173]$ utilize metal heaters and the temperature is accurately determined from the resistance value of the heating element by using a separate pair of electrodes in a four-point probe measurement. Precise temperature stabilization $\left(<0.1^{\circ} \mathrm{C} / \mathrm{min}\right)$ is achieved by continuously monitoring the resistance in a closed control loop, hence guaranteeing accuracy and reproducibility of the in situ experiments. Thanks to the localized heating and the small thermal mass involved, low power consumption (few tens of milliwatts) is achieved enabling temperatures as high as $1300^{\circ} \mathrm{C}$, heating and cooling in the millisecond range, minor sample displacement, and drift stabilization within few minutes instead of hours. Thus, with some care heat-induced specimen modifications can be recorded at atomic resolution in real time from the first instance after a heat change [174-176].

\subsection{Electrical stages}

In early studies, instruments and attachments for in situ biasing TEM were laboratory-made systems [177-183]. Practically, these experiments required complicated sample preparation strategies and it was difficult to estimate the magnitude of the actual electric field applied to the imaging area of the specimen. In the early 2000s, the commercialization by Nanofactory Instruments of TEM holders with an integrated scanning probe made in situ biasing experiments much easier [184-188]. These in situ holders use a conductive nanoprobe that is brought into contact to the specimen by using a precise piezo-driven manipulator. In particular, for polarization switching experiments of ferroelectric thin films, cross-sectional specimens are required. Thus, an electric field can be induced along the film normal by applying a voltage between the nanoprobe (contacted at the film surface) and a bottom electrode (contacted with silver paste at the conductive substrate or buffer layer); see Figure 20(a). A typical ferroelectric switching process observed in situ using a conductive nanoprobe is shown in Figure 20(b and c). In this example, the nucleation and growth of a $\mathrm{P}[00-1]$ domain in a $\mathrm{PbZr}_{0.2} \mathrm{Ti}_{0.8} \mathrm{O}_{3}$ film is observed to take place in the region below the tip of the probe during the initial switching cycle, while reverse switching under reverse bias results in the nucleation of multiple P[001] domains at the bottom interface [189]. While much research on the switching mechanisms has been conducted by using in situ conductive nanoprobe systems [184, 187-190], they face two serious disadvantages. First, it is not possible to examine ferroelectric switching over large areas under a uniform electric field. Instead, an inhomogeneous high field is confined to the region below the tip of the probe [187-189]. Second, additional piezoelectric and flexoelectric coupling effects on the spontaneous polarization might arise from the force contact of the probe against the film surface [191, 192]. This suggests that the employed geometry, where the film surface is in direct contact with the probe, is probably not entirely reliable. As an alternative, a sandwich-type capacitor geometry with bottom and top electrodes would be a better choice [193, 194]. Figure 21 shows the switching behavior of an epitaxial $\mathrm{PbZr}_{0.2} \mathrm{Ti}_{0.8} \mathrm{O}_{3}$ thin film sandwiched between a conductive $\mathrm{Nb}-\mathrm{SrTiO}_{3}$ substrate and a top electrode consisting of $\mathrm{Au}$ and $\mathrm{Pt}$ in a capacitor-type geometry [193]. The series of dark-field TEM images acquired at distinct external biases demonstrates that by using this configuration a uniform electric field is attained over a very large area. Moreover, this geometry ensures that the electrical boundary conditions closely resemble those of an actual ferroelectric capacitor [195]. Here, also, advances in MEMS technology for miniaturized TEM specimen holders shall bring new opportunities for more consistent in situ biasing studies. Similarly to the MEMS heating chips, this approach uses prefabricated circuitry developed by lithography on silicon chips containing thin silicon nitride membranes. A cross-sectional slice of the ferroelectric film sandwiched between two conductive layers is prepared by FIB. Then, the conductive layers are connected to the circuitry by writing metal interconnects by electron or ion-beam-induced deposition within a SEM or FIB [196]. Notably, MEMS-based holders 
and chips fitted with four or more contacts offer the unique additional capability to enable simultaneous in situ heating and biasing experiments, thus, opening up new fields of research for in situ electron microscopy.

(a)
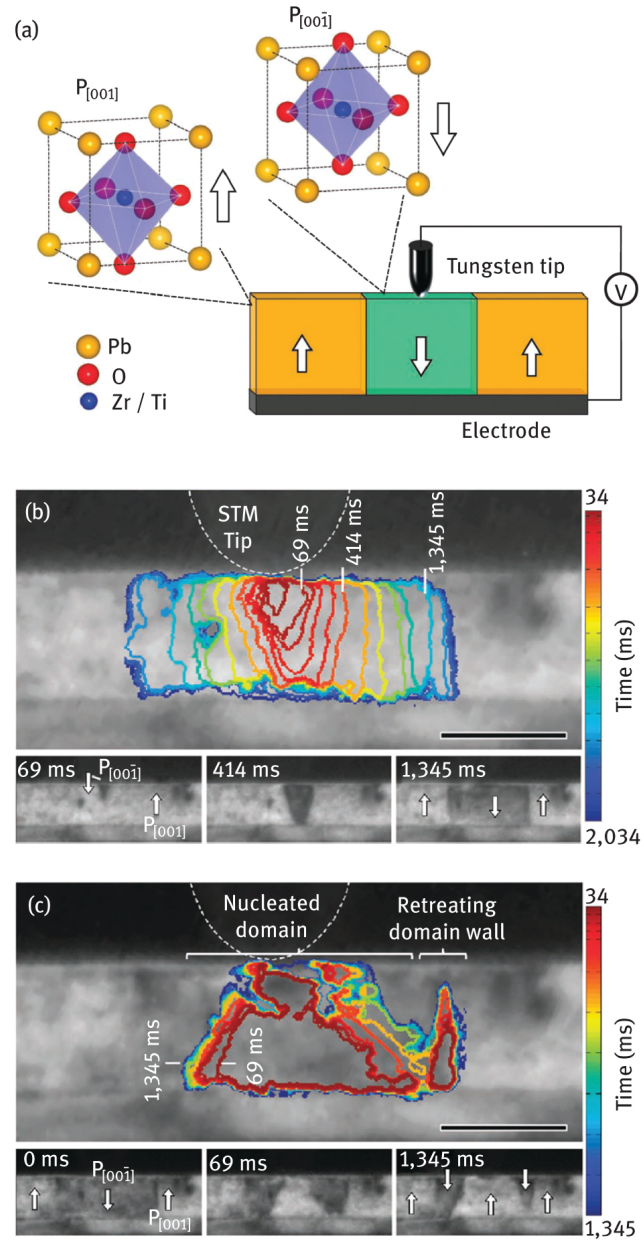

Figure 20: In situ TEM observations of polarization switching using a conductive nanoprobe. (a) Schematics of the switching experiment in the TEM. Polarization switching is induced by an applied electric field oriented along the film normal between a surface probe in direct contact with the $\mathrm{PbZr}_{0.2} \mathrm{Ti}_{0.8} \mathrm{O}_{3}$ film surface and a planar bottom electrode. (b) Growth of a P[00-1] domain during the initial switching cycle. (c) Reverse switching under reverse bias results in the nucleation of P[001] domains at the bottom. The lines are contour plots of the domain outline versus time. Selected corresponding TEM images are shown below each panel. Scale bars are $100 \mathrm{~nm}$. Reproduced from [189]. 


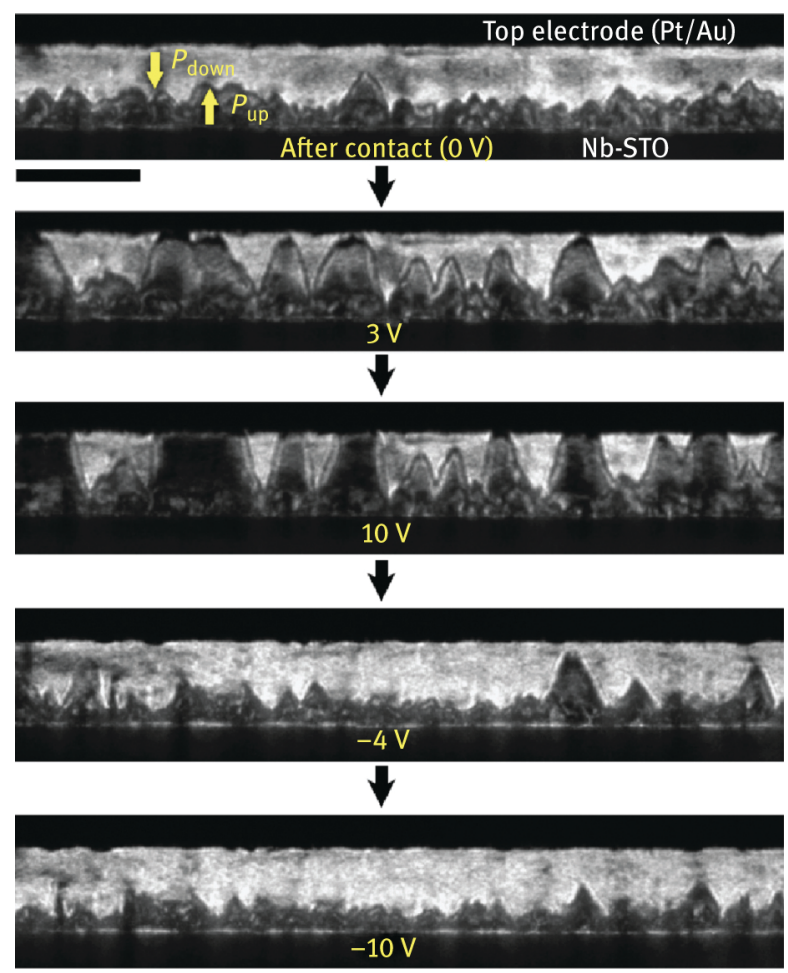

Figure 21: In situ TEM observations of polarization switching using a capacitor-type geometry. The ferroelectric $\mathrm{PbZr}_{0.2} \mathrm{Ti}_{0.8} \mathrm{O}_{3}$ thin film is sandwiched between a conductive $\mathrm{Nb}-\mathrm{SrTiO}_{3}$ substrate and a top electrode consisting of $\mathrm{Au}$ and Pt. The series of dark-field TEM images show the domain-switching behavior induced by a series of external biases. The scale bar is $200 \mathrm{~nm}$. Reproduced from [193].

For all the reasons explained above, MEMS-based TEM heating holders are becoming increasingly popular. This is reflected in the exponential growth in the number of publications, and material conference sessions and workshops on in situ TEM organized over the last decade. In particular, it is expected that the MEMS-based in situ technology shall become standard for characterizing ferroelectric materials enabling a whole new level of insight into multiferroic properties at unprecedented length scales. For example, in situ thermal TEM experiments shall be used to study the ferroelectric phase transition in thin films and to investigate the mechanisms controlling the Curie temperature in the low thickness range, such as clamping of the film distortions exerted by the substrate. These experiments performed at the atomic scale should give direct insight into the ferroelectric ordering and help clarify the displacement and order-disorder polarization mechanisms. Dynamic measurements of the polarization reversal by applying electric fields through the connected top and bottom electrodes will be crucial to study in situ the polarization switching process and domain wall propagation at the atomic scale. In situ electron holography, at forward and reverse bias conditions, shall reveal valued information about the charge redistribution. Also, in situ biasing experiments shall allow for observing the response of magnetic domains to the applied electric field, which is essential to understanding the multiferroic behavior. In conclusion, MEMS-based TEM holders shall contribute significantly to understanding the relationship between the domain dynamics of multiferroics and the specific microstructure of the films providing important guidance to design new devices and to predict and mitigate failures.

\section{Summary}

This review focused on introducing advanced methods based on (scanning) transmission electron microscopy which can be used to characterize complex multiferroic materials. The review did not deal with basic TEM methods, such as bright-field/dark-field imaging, selected area diffraction, which, of course, are important for the study of materials, in general, and undoubtedly need to be considered as the fundament upon which new and advanced methods build up. Neither did the review deal with analytical methods, such as EELS and EDXS, as the focus of the present review was on analytical imaging methods. After introducing the topic, so-called phase-contrast methods were first discussed which can be used to map electrostatic and magnetic fields in TEM and STEM modus. Diffraction methods were then covered which provide very local diffraction information; these are particularly useful to gather structural data in materials consisting of small domains. Thereafter, methods were discussed which allow for analyzing local strain, whereas in the subsequent section 
methods were introduced which allow for measuring local polarization effects on a length scale of individual unit cells. Finally, a brief outlook was given about the possibilities of new in situ methods which are just about at the launch of becoming more popular, particularly in the field of magnetoelectric multiferroics.

\section{References}

[1] Erni R. Aberration-corrected imaging in transmission electron microscopy. London: Imperial College Press, 2014.

[2] Carter CB, Williams DB. Transmission electron microscopy: diffraction, imaging, and spectrometry. Switzerland: Springer International Publishing, 2016

[3] Ramasse QM, Seabourne CR, Kepaptsoglou D-M, Zan R, Bangert U, Scott A]. Probing the bonding and electronic structure of single atom dopants in graphene with electron energy loss spectroscopy. Nano Lett. 2013;13:4989.

[4] Williams DB, Carter CB. Transmission electron microscopy. United States: Springer, 2009.

[5] Newnham RE. Properties of materials: anisotropy, symmetry, structure. New York, United States: Oxford University Press, 2005

[6] Fiebig M, Lottermoser T, Meier D, Trassin M. The evolution of multiferroics. Nat Rev Mater. 2016;1:16046.

[7] Matteucci G, Missiroli G, Nichelatti E, Migliori A, Vanzi M, Pozzi G. Electron holography of long-range electric and magnetic fields. ] Appl Phys. 1991;69:1835.

[8] Rau WD, Schwander P, Baumann FH, Höppner W, Ourmazd A. Two-dimensional mapping of the electrostatic potential in transistors by electron holography. Phys Rev Lett. 1999;82:2614.

[9] Cumings ], Zettl A, McCartney MR, Spence ]C. Electron holography of field-emitting carbon nanotubes. Phys Rev Lett. 2002;88:056804.

[10] Kasama T, Dunin-Borkowski RE, Beleggia M. Electron holography of magnetic materials. In: Monroy F, editor(s). Holography-Different fields of application, 2011 ed. London, United Kingdom: IntechOpen.

[11] Nagaosa N, Tokura Y. Topological properties and dynamics of magnetic skyrmions. Nat Nanotechnol. 2013;8:899.

[12] Pollard SD, Garlow JA, Yu ], Wang Z, Zhu Y, Yang H. Observation of stable Néel skyrmions in cobalt/palladium multilayers with Lorentz transmission electron microscopy. Nat Commun. 2017;8:1.

[13] Phatak C, Petford-Long AK, Heinonen O. Direct observation of unconventional topological spin structure in coupled magnetic discs. Phys Rev Lett. 2012;108:067205.

[14] Rubiano Da Silva N, Möller M, Feist A, Ulrichs H, Ropers C, Schäfer S. Nanoscale mapping of ultrafast magnetization dynamics with femtosecond Lorentz microscopy. Phys Rev X. 2018;8:31052.

[15] Fu X, Pollard SD, Chen B, Yoo BK, Yang H, Zhu Y. Optical manipulation of magnetic vortices visualized in situ by Lorentz electron microscopy. Sci Adv. 2018;4:eaat3077.

[16] Rodríguez LA, Magén C, Snoeck E, Gatel C, Marín L, Serrano-Ramón L, et al. Quantitative in situ magnetization reversal studies in Lorentz microscopy and electron holography. Ultramicroscopy. 2013;134:144.

[17] Shibata N, Kohno Y, Nakamura A, Morishita S, Seki T, Kumamoto A, et al. Atomic resolution electron microscopy in a magnetic field free environment. Nat Commun. 2019;10:2308.

[18] Aharonov Y, Bohm D. Significance of electromagnetic potentials in the quantum theory. Phys Rev. 1959;115:485.

[19] Winkler F, Tavabi AH, Barthel ], Duchamp M, Yucelen E, Borghardt S, et al. Quantitative measurement of mean inner potential and specimen thickness from high-resolution off-axis electron holograms of ultra-thin layered WSe2. Ultramicroscopy. 2017;178:38.

[20] Lichte H, Reibold M, Brand K, Lehmann M. Ferroelectric electron holography. Ultramicroscopy. 2002;93:199.

[21] Beleggia M, Kasama T, Dunin-Borkowski RE. The quantitative measurement of magnetic moments from phase images of nanoparticles and nanostructures-I. Fundamentals. Ultramicroscopy. 2010;110:425.

[22] Reimer L, Kohl H. Scattering and phase-contrast. Springer Ser Opt Sci. 2008;36:193.

[23] Bonevich]E, Pozzi G, Tonomura A. Electron holography of electromagnetic fields. Introduction to electron holography. Boston, MA, United States: Springer, 1999.

[24] Lichte H, Lehmann M. Electron holography—basics and applications. Reports Prog Phys. 2008;71:016102.

[25] Möllenstedt C, Düker H. Beobachtungen und Messungen an Biprisma-Interferenzen mit Elektronenwellen. Zeitschrift Für Phys. 1956;145:377.

[26] Völkl E, Lehmann M. The reconstruction of off-axis electron holograms. Introduction to electron holography. Boston, MA, United States: Springer, 1999.

[27] Lehmann M, Lichte H. Tutorial on off-axis electron holography. Microsc Microanal. 2002;8:447.

[28] Röder F, Lubk A, Wolf D, Niermann T. Noise estimation for off-axis electron holography. Ultramicroscopy. 2014;144:32.

[29] Frost BC, Matteucci C. On recording, processing and interpretation of low magnification electron holograms. Introduction to electron holography. Boston, MA, United States: Springer, 1999.

[30] de Ruijter W], Weiss ]K. Detection limits in quantitative off-axis electron holography. Ultramicroscopy. 1993;50:269.

[31] Cooper D, Truche R, Rivallin P, Hartmann J-M, Laugier F, Bertin F, et al. Medium resolution off-axis electron holography with millivolt sensitivity. Appl Phys Lett. 2007;91:143501.

[32] Harscher A, Lichte H. Experimental study of amplitude and phase detection limits in electron holography. Ultramicroscopy. 1996;64:57.

[33] Phatak C, Bain JA, Zhu JG, De Graef M. Aberration corrected Lorentz microscopy for perpendicular magnetic recording media. Microsc Microanal. 2008;14:832.

[34] Lehmann M, Lichte H, Geiger D, Lang C, Schweda E. Electron holography at atomic dimensions—present state. Mater Charact. 1999;42:249.

[35] Lichte H. Electron image plane off-axis holography of atomic structures. In: Mulvey T, Sheppard C, editor(s). Advances in optical and electron microscopy Vol. 12. San Diego, CA, United States: Academic Press Inc, 1991:25. 
[36] Rau WD, Lichte H. High resolution off-axis electron holography. Introduction to electron holography. Boston, MA, United States: Springer, 1999.

[37] Tonomura A, Matsuda T, Endo ], Arii T, Mihama K. Holographic interference electron microscopy for determining specimen magnetic structure and thickness distribution. Phys Rev B. 1986;34:3397.

[38] Kasama T, Dunin-Borkowski RE, Scheinfein MR, Tripp SL, Liu ], Wei A. Off-axis electron holography of self-assembled Co nanoparticle rings. MRS Proc. 2007;1026:1003.

[39] Lazić I, Bosch EC. Analytical review of direct STEM imaging techniques for thin samples. Adv Imaging Electron Phys. 2017;199:75.

[40] Rose H. Phase-contrast in scanning transmission electron microscopy. Optik (Stuttg). 1974;39:416.

[41] Dekkers NH, Lang HD. Differential phase-contrast in a STEM. Optik (Stuttg). 1974;41:452.

[42] Erni R, Rossell MD, Kisielowski C, Dahmen U. Atomic-resolution imaging with a sub-50-pm electron probe. Phys Rev Lett. 2009;102:96101.

[43] Chapman JN, Batson PE, Waddell EM, Ferrier RP. The direct determination of magnetic domain wall profiles by differential phasecontrast electron microscopy. Ultramicroscopy. 1978;3:203.

[44] Chapman JN, Morrison GR, Jakubovics ]P, Taylor RA. Determination of domain wall structures in thin foils of a soft magnetic alloy. ] Magn Magn Mater. 1985;49:277.

[45] Sannomiya T, Haga Y, Nakamura Y, Nittono O, Takahashi Y. Observation of magnetic structures in Fe granular films by differential phasecontrast scanning transmission electron microscopy. J Appl Phys. 2004;95:214.

[46] O'Shea K], MacLaren DA, McGrouther D, Schwarzbach D, Jungbauer M, Hühn S, et al. Nanoscale mapping of the magnetic properties of (111)-oriented Lao.67Sro.33MnO3. Nano Lett. 2015;15:5868.

[47] Chen C, Li H, Seki T, Yin D, Sanchez-Santolino G, Inoue K, et al. Direct determination of atomic structure and magnetic coupling of magnetite twin boundaries. ACS Nano. 2018;12:2662.

[48] Brown HC, Shibata N, Sasaki H, Petersen TC, Paganin DM, Morgan M], et al. Measuring nanometre-scale electric fields in scanning transmission electron microscopy using segmented detectors. Ultramicroscopy. 2017;182:169.

[49] Haas B, Rouvière ]-L, Boureau V, Berthier R, Cooper D. Direct comparison of off-axis holography and differential phase-contrast for the mapping of electric fields in semiconductors by transmission electron microscopy. Ultramicroscopy. 2019;198:58.

[50] Shibata N, Findlay SD, Sasaki H, Matsumoto T, Sawada H, Kohno Y, et al. Imaging of built-in electric field at a p-n junction by scanning transmission electron microscopy. Sci Rep. 2015;5:10040.

[51] Shibata N, Findlay SD, Kohno Y, Sawada H, Kondo Y, Ikuhara Y. Differential phase-contrast microscopy at atomic resolution. Nat Phys. 2012;8:611.

[52] Shibata N, Seki T, Sánchez-Santolino G, Findlay SD, Kohno Y, Matsumoto T, et al. Electric field imaging of single atoms. Nat Commun. 2017;8:15631.

[53] Campanini M, Ciprian R, Bedogni E, Mega A, Chiesi V, Casoli F, et al. Lorentz microscopy sheds light on the role of dipolar interactions in magnetic hyperthermia. Nanoscale. 2015;7:7717.

[54] Hachtel ]A, Idrobo JC, Chi M. Sub-Ångstrom electric field measurements on a universal detector in a scanning transmission electron microscope. Adv Struct Chem Imaging. 2018;4:10.

[55] Krajnak M, McGrouther D, Maneuski D, O'Shea V, McVitie S. Pixelated detectors and improved efficiency for magnetic imaging in STEM differential phase-contrast. Ultramicroscopy. 2016;165:42.

[56] Campanini M, Erni R, Yang CH, Ramesh R, Rossell MD. Periodic giant polarization gradients in doped BiFeO3 thin films. Nano Lett. 2018;18:717.

[57] Shibata N, Findlay SD, Matsumoto T, Kohno Y, Seki T, Sánchez-Santolino G, et al. Direct visualization of local electromagnetic field structures by scanning transmission electron microscopy. Acc Chem Res. 2017;50:1502.

[58] Zweck ], Schwarzhuber F, Wild ], Galioit V. On detector linearity and precision of beam shift detection for quantitative differential phasecontrast applications. Ultramicroscopy. 2016;168:53.

[59] Close R, Chen Z, Shibata N, Findlay SD. Towards quantitative, atomic-resolution reconstruction of the electrostatic potential via differential phase-contrast using electrons. Ultramicroscopy. 2015;159:124.

[60] Müller K, Krause FF, Béché A, Schowalter M, Galioit V, Löffler S, et al. Atomic electric fields revealed by a quantum mechanical approach to electron picodiffraction. Nat Commun. 2014;5:5653.

[61] Ehrenfest P. Bemerkung über die angenäherte Cültigkeit der klassischen Mechanik innerhalb der Quantenmechanik. Zeitschrift Für Phys. 1927;45:455.

[62] Yang H, Jones L, Ryll H, Simson M, Soltau H, Kondo Y, et al. 4D STEM: high efficiency phase-contrast imaging using a fast pixelated detector. J Phys Conf Ser. 2015;644:012032.

[63] Chen Z, Weyland M, Ercius P, Ciston ], Zheng C, Fuhrer MS, et al. Practical aspects of diffractive imaging using an atomic-scale coherent electron probe. Ultramicroscopy. 2016;169:107.

[64] Fang S, Wen Y, Allen CS, Ophus C, Han CG, Kirkland AI, et al. Atomic electrostatic maps of 1D channels in 2D semiconductors using 4D scanning transmission electron microscopy. Nat Commun. 2019;10:1127.

[65] Jesse S, Chi M, Belianinov A, Beekman C, Kalinin SV, Borisevich AY, et al. Big data analytics for scanning transmission electron microscopy ptychography. Sci Rep. 2016;6:26348.

[66] Belianinov A, Vasudevan R, Strelcov E, Steed C, Yang SM, Tselev A, et al. Big data and deep data in scanning and electron microscopies: deriving functionality from multidimensional data sets. Adv Struct Chem Imaging. 2015;1:6.

[67] Tate MW, Purohit P, Chamberlain D, Nguyen KX, Hovden R, Chang CS, et al. High dynamic range pixel array detector for scanning transmission electron microscopy. Microsc Microanal. 2016;22:237.

[68] Faruqi AR, McMullan C. Direct imaging detectors for electron microscopy. Nucl Instrum Methods Phys Res A. 2018;878:180.

[69] Lubk A, Zweck ]. Differential phase-contrast: an integral perspective. Phys Rev A. 2015;91:023805.

[70] Lubk A, Wolf D, Lichte $H$. The effect of dynamical scattering in off-axis holographic mean inner potential and inelastic mean free path measurements. Ultramicroscopy. 2010;110:438. 
[71] Rose H. Nonstandard imaging methods in electron microscopy. Ultramicroscopy. 1976;2:251.

[72] Shindo D, Murakami Y. Electron holography of magnetic materials. ] Phys D: Appl Phys. 2008;41:183002.

[73] Matsumoto T, Koguchi M, Suzuki K, Nishimura H, Motoyoshi Y, Wada N. Ferroelectric $90^{\circ}$ domain structure in a thin film of BaTiO3 fine ceramics observed by $300 k V$ electron holography. Appl Phys Lett. 2008;92:72902.

[74] Polking M], Han M-G, Yourdkhani A, Petkov V, Kisielowski CF, Volkov VV. Ferroelectric order in individual nanometre-scale crystals. Nat Mater. 2012;11:700.

[75] Chapman JN. The investigation of magnetic domain structures in thin foils by electron microscopy. J Phys D Appl Phys. 1984;17:623.

[76] Körner M, Röder F, Lenz K, Fritzsche M, Lindner ], Lichte H, et al. Quantitative imaging of the magnetic configuration of modulated nanostructures by electron holography. Small. 2014;10:5161.

[77] Ranzieri P, Campanini M, Fabbrici S, Nasi L, Casoli F, Cabassi R, et al. Achieving giant magnetically induced reorientation of martensitic variants in magnetic shape-memory Ni-Mn-Ga films by microstructure engineering. Adv Mater. 2015;27:4760.

[78] Gatel C, Warot-Fonrose B, Biziere N, Rodríguez LA, Reyes D, Cours R, et al. Inhomogeneous spatial distribution of the magnetic transition in an iron-rhodium thin film. Nat Commun. 2017;8:15703.

[79] Agrawal P, Campanini M, Rappe A, Liu S, Grillo V, Hébert C, et al. Structure and properties of edge dislocations in BiFeO3. Phys Rev Mater. 2019;3:034410.

[80] Akhtari-Zavareh A, Carignan LP, Yelon A, Ménard D, Kasama T, Herring R, et al. Off-axis electron holography of ferromagnetic multilayer nanowires. J Appl Phys. 2014;116:23902.

[81] Cantu-Valle ], Díaz Barriga-Castro E, Vega V, García ], Mendoza-Reséndez R, Luna C, et al. Quantitative magnetometry analysis and structural characterization of multisegmented cobalt-nickel nanowires. J Magn Magn Mater. 2015;379:294.

[82] Reyes D, Biziere N, Warot-Fonrose B, Wade T, Gatel C. Magnetic configurations in Co/Cu multilayered nanowires: evidence of structural and magnetic interplay. Nano Lett. 2016;16:1230.

[83] Rodríguez LA, Bran C, Reyes D, Berganza E, Vázquez M, Gatel C, et al. Quantitative nanoscale magnetic study of isolated diametermodulated FeCoCu nanowires. ACS Nano. 2016;10:9669.

[84] Snoeck E, Gatel C, Lacroix LM, Blon T, Lachaize S, Carrey ], et al. Magnetic configurations of $30 \mathrm{~nm}$ iron nanocubes studied by electron holography. Nano Lett. 2008;8:4293.

[85] Gatel C, Bonilla F], Meffre A, Snoeck E, Warot-Fonrose B, Chaudret B, et al. Size-specific spin configurations in single iron nanomagnet: from flower to exotic vortices. Nano Lett. 2015;15:6952.

[86] Varón M, Beleggia M, Kasama T, Harrison R], Dunin-Borkowski RE, Puntes VF, et al. Dipolar magnetism in ordered and disordered lowdimensional nanoparticle assemblies. Sci Rep. 2013;3:3.

[87] Zuo ]-M, Tao ]. Scanning electron nanodiffraction and diffraction imaging. Scanning transmission electron microscopy. New York: Springer, 2011.

[88] Zuo ]M, Spence ]C. Advanced transmission electron microscopy. New York: Springer, 2017.

[89] Kuhn M, Cea S, Zhang ], Wormington M, Nuytten T, De Wolf I, et al. Transistor strain measurement techniques and their applications. In: Ma Z, Seiler DC, editor(s). Metrology and diagnostic techniques for nanoelectronics. New York, United States: Pan Stanford Ltd., 2016.

[90] Gao M, Zuo ]M, Twesten RD, Petrov I, Nagahara LA, Zhang R. Structure determination of individual single-wall carbon nanotubes by nanoarea electron diffraction. Appl Phys Lett. 2003;82:2703.

[91] Zuo ]M, Gao M, Tao ], Li BQ, Twesten R, Petrov I. Coherent nano-area electron diffraction. Microsc Res Tech. 2004;64:347.

[92] Armigliato A, Frabboni S, Gazzadi CC. Electron diffraction with ten nanometer beam size for strain analysis of nanodevices. Appl Phys Lett. 2008;93:161906.

[93] Béché A, Rouvière ]L, Clément L, Hartmann JM. Improved precision in strain measurement using nanobeam electron diffraction. Appl Phys Lett. 2009;95:123114.

[94] Chen LC, Chen FR, Kai J], Chang L, Ho ]K, Jong CS, et al. Microstructural investigation of oxidized Ni/Au ohmic contact to p-type GaN. ] Appl Phys. 1999;86:3826.

[95] Okamoto H, Hokazono A, Adachi K, Yasutake N, Itokawa H, Okamoto S, et al. In situ doped embedded-SiCe source/drain technique for 32 nm node p-channel metal-oxide-semiconductor field-effect transistor. Jpn ] Appl Phys. 2008;47:2564.

[96] Toda A, Nakamura H, Fukai T, Ikarashi N. Channel strain in advanced complementary metal-oxide-semiconductor field effect transistors measured using nano-beam electron diffraction. Jpn ] Appl Phys. 2008;47:2496.

[97] Favia P, Popovici M, Eneman G, Wang G, Bargallo-Conzalez M, Simoen E, et al. Nano-beam diffraction: crystal structure and strain analysis at the nanoscale. ECS Transactions. 2010;33:205

[98] Kim SW, Byeon DS, Jang H, Koo SM, Lee H], Ko DH. Strain characterization of fin-shaped field effect transistors with SiCe stressors using nanobeam electron diffraction. Appl Phys Lett. 2014;105:83104.

[99] Cooper D, Denneulin T, Bernier N, Béché A, Rouvière ]L. Strain mapping of semiconductor specimens with nm-scale resolution in a transmission electron microscope. Micron. 2016;80:145.

[100] Vincent R, Midgley PA. Double conical beam-rocking system for measurement of integrated electron diffraction intensities. Ultramicroscopy. 1994;53:271.

[101] Miao S, Zhang X, Zhu ]. Direct compositional analysis of ordered domains in pure and La-Doped Pb(Mg1/3Nb2/3)O3 by analytical electron microscopy techniques. ] Am Ceram Soc. 2004;84:2091.

[102] Béché A, Rouvière ]L, Barnes ]P, Cooper D. Strain measurement at the nanoscale: comparison between convergent beam electron diffraction, nano-beam electron diffraction, high resolution imaging and dark field electron holography. Ultramicroscopy. 2013;131:10.

[103] Hÿtch M, Houdellier F, Hüe F, Snoeck E. Nanoscale holographic interferometry for strain measurements in electronic devices. Nature. 2008;453:1086.

[104] Hÿtch M], Houdellier F, Hüe F, Snoeck E. Dark-field electron holography for the measurement of geometric phase. Ultramicroscopy. 2011;111:1328.

[105] Denneulin T, Cooper D, Rouvière ]L. Practical aspects of strain measurement in thin SiCe layers by (004) dark-field electron holography in Lorentz mode. Micron. 2014;62:52. 
[106] Béché A, Rouvière ]L, Barnes ]P, Cooper D. Dark field electron holography for strain measurement. Ultramicroscopy. 2011;111:227.

[107] Hüe F, Hÿtch M, Houdellier F, Snoeck E, Claverie A. Strain mapping in MOSFETS by high-resolution electron microscopy and electron holography. Mater Sci Eng B Solid-State Mater Adv Technol. 2008;154-155:221.

[108] Cooper D, Barnes JP, Hartmann JM, Béché A, Rouvière JL. Dark field electron holography for quantitative strain measurements with nanometer-scale spatial resolution. Appl Phys Lett. 2009;95:53501.

[109] Denneulin T, Wollschläger N, Everhardt AS, Farokhipoor S, Noheda B, Snoeck E, et al. Local deformation gradients in epitaxial $\mathrm{Pb}\left(\mathrm{Zr}_{0.2} \mathrm{Ti}_{0.8}\right) \mathrm{O}_{3}$ layers investigated by transmission electron microscopy. J Phys Condens Matter. 2018;30:215701.

[110] Pennycook S]. Scanning transmission electron microscopy: Z-contrast imaging. Characterization of materials. New Jersey, United States: Wiley, 2012.

[111] Jones L, Wenner S, Nord M, Ninive PH, Løvvik OM, Holmestad R, et al. Optimising multi-frame ADF-STEM for high-precision atomicresolution strain mapping. Ultramicroscopy. 2017;179:57.

[112] Sanchez AM, Galindo PL, Kret S, Falke M, Beanland R, Goodhew P]. Quantitative strain mapping applied to aberration-corrected HAADF images. Microsc Microanal. 2006;12:285.

[113] Sanchez AM, Galindo PL, Kret S, Falke M, Beanland R, Goodhew P]. An approach to the systematic distortion correction in aberrationcorrected HAADF images. ] Microsc. 2006;221:1.

[114] Zuo JM, Shah AB, Kim H, Meng Y, Gao W, Rouvière JL. Lattice and strain analysis of atomic resolution Z-contrast images based on template matching. Ultramicroscopy. 2014;136:50.

[115] Lubk A, Rossell MD, Seidel ], He Q, Yang SY, Chu YH, et al. Evidence of sharp and diffuse domain walls in BiFeO3 by means of unitcell-wise strain and polarization maps obtained with high resolution scanning transmission electron microscopy. Phys Rev Lett. 2012;109:047601.

[116] Couillard M, Radtke G, Botton CA. Strain fields around dislocation arrays in a $\Sigma 9$ silicon bicrystal measured by scanning transmission electron microscopy. Philos Mag. 2013;93:1250.

[117] Sang X, LeBeau ]M. Revolving scanning transmission electron microscopy: correcting sample drift distortion without prior knowledge. Ultramicroscopy. 2014;138:28.

[118] Yankovich AB, Berkels B, Dahmen W, Binev P, Sanchez SI, Bradley SA, et al. Picometre-precision analysis of scanning transmission electron microscopy images of platinum nanocatalysts. Nat Commun. 2014;5:4155.

[119] Jones L, Yang H, Pennycook T], Marshall MS, Van Aert S, Browning ND, et al. Smart Align-a new tool for robust non-rigid registration of scanning microscope data. Adv Struct Chem Imaging. 2015;1:8

[120] Hÿtch M], Snoeck E, Kilaas R. Quantitative measurement of displacement and strain fields from HREM micrographs. Ultramicroscopy. 1998;74:131.

[121] Hÿtch M], Putaux ]-L, Pénisson ]-M. Measurement of the displacement field of dislocations to $0.03 \AA$ by electron microscopy. Nature. 2003;423:270.

[122] Johnson CL, Hÿtch M], Buseck PR. Nanoscale waviness of low-angle grain boundaries. Proc Natl Acad Sci. 2004;101:17936.

[123] Du K, Rau Y, Jin-Phillipp NY, Phillipp F. Lattice distortion analysis directly from high resolution transmission electron microscopy images—the \{LADIA\} program package. ] Mater Sci. 2002;18:135.

[124] Galindo PL, Kret S, Sanchez AM, Laval JY, Yáñez A, Pizarro ], et al. The Peak Pairs algorithm for strain mapping from HRTEM images. Ultramicroscopy. 2007;107:1186.

[125] Galindo P, Pizarro ], Molina S, Ishizuka K. High resolution peak measurement and strain mapping using peak pairs analysis. Microsc Anal. 2009;23:23.

[126] Rossell MD, Erni R, Prange MP, Idrobo ]-C, Luo W, Zeches R], et al. Atomic structure of highly strained BiFeO3 thin films. Phys Rev Lett. 2012;108:047601.

[127] Zeches R], Rossell MD, Zhang JX, Hatt A], He Q, Yang C-H, et al. A strain-driven morphotropic phase boundary in BiFeO3. Science. 2009;326:977.

[128] Agrawal P, Guo ], Yu P, Hébert C, Passerone D, Erni R, et al. Strain-driven oxygen deficiency in multiferroic SrMnO3 thin films. Phys Rev B. 2016;94:104101.

[129] Zhang ]X, He Q, Trassin M, Luo W, Yi D, Rossell MD, et al. Microscopic origin of the giant ferroelectric polarization in tetragonal-like BiFeO3. Phys Rev Lett. 2011;107:147602.

[130] Guo ]W, Wang PS, Yuan Y, He Q, Lu JL, Chen TZ, et al. Strain-induced ferroelectricity and spin-lattice coupling in SrMnO3 thin films. Phys Rev B. 2018;97:235135.

[131] Jia CL, Urban KW, Alexe M, Hesse D, Vrejoiu I. Direct observation of continuous electric dipole rotation in flux-closure domains in ferroelectric $\mathrm{Pb}(\mathrm{Zr}, \mathrm{Ti}) \mathrm{O} 3$. Science. 2011;331:1420.

[132] Wang WY, Tang YL, Zhu YL, Xu YB, Liu Y, Wang Y], et al. Atomic level 1D structural modulations at the negatively charged domain walls in BiFeO3 films. Adv Mater Interfaces. 2015;2:1500024.

[133] Lubk A, Rossell MD, Seidel ], Chu YH, Ramesh R, Hÿtch M], et al. Electromechanical coupling among edge dislocations, domain walls, and nanodomains in $\mathrm{BiFeO}_{3}$ revealed by unit-cell-wise strain and polarization maps. Nano Lett. 2013;13:1410.

[134] Li L, Gao P, Nelson CT, Jokisaari ]R, Zhang Y, Kim S], et al. Atomic scale structure changes induced by charged domain walls in ferroelectric materials. Nano Lett. 2013;13:5218.

[135] De Luca G, Strkalj N, Manz S, Bouillet C, Fiebig M, Trassin M. Nanoscale design of polarization in ultrathin ferroelectric heterostructures. Nat Commun. 2017;8:1419.

[136] Tang YL, Zhu YL, Ma XL, Borisevich AY, Morozovska AN, Eliseev EA, et al. Observation of a periodic array of flux-closure quadrants in strained ferroelectric $\mathrm{PbTiO}_{3}$ films. Science. 2015;348:547.

[137] Nelson CT, Winchester B, Zhang Y, Kim S], Melville A, Adamo C, et al. Spontaneous vortex nanodomain arrays at ferroelectric heterointerfaces. Nano Lett. 2011;11:828.

[138] Yadav AK, Nelson CT, Hsu SL, Hong Z, Clarkson JD, Schlepuëtz CM, et al. Observation of polar vortices in oxide superlattices. Nature. 2016;530:198. 
[139] Gao P, Liu H], Huang YL, Chu YH, Ishikawa R, Feng B, et al. Atomic mechanism of polarization-controlled surface reconstruction in ferroelectric thin films. Nat Commun. 2016;7:11318.

[140] Campanini M, Trassin M, Ederer C, Erni R, Rossell MD. Buried in-plane ferroelectric domains in Fe-doped single-crystalline Aurivillius thin films. ACS Appl Electron Mater. 2019;1:1019.

[141] Damodaran AR, Pandya S, Qi Y, Hsu SL, Liu S, Nelson C, et al. Large polarization gradients and temperature-stable responses in compositionally-graded ferroelectrics. Nat Commun. 2017;8:14961.

[142] Jin L, Xu PX, Zeng Y, Lu L, Barthel ], Schulthess T, et al. Surface reconstructions and related local properties of a BiFeO3 thin film. Sci Rep. 2017;7:39698.

[143] Okunishi E, Ishikawa I, Sawada H, Hosokawa F, Hori M, Kondo Y. Visualization of light elements at ultrahigh resolution by STEM annular bright field microscopy. Microsc Microanal. 2009;15:164.

[144] Ishikawa R, Okunishi E, Sawada H, Kondo Y, Hosokawa F, Abe E. Direct imaging of hydrogen-atom columns in a crystal by annular bright-field electron microscopy. Nat Mater. 2011;10:278.

[145] Oshima Y, Sawada H, Hosokawa F, Okunishi E, Kaneyama T, Kondo Y, et al. Direct imaging of lithium atoms in LiV2O4 by spherical aberration-corrected electron microscopy.] Jlectron Microsc (Tokyo). 2010;59:457.

[146] Findlay SD, Shibata N, Sawada H, Okunishi E, Kondo Y, Yamamoto T, et al. Robust atomic resolution imaging of light elements using scanning transmission electron microscopy. Appl Phys Lett. 2009;95:191913.

[147] Findlay SD, Shibata N, Sawada H, Okunishi E, Kondo Y, Ikuhara Y. Dynamics of annular bright field imaging in scanning transmission electron microscopy. Ultramicroscopy. 2010;110:903.

[148] Findlay SD, Saito T, Shibata N, Sato Y, Matsuda ], Asano K, et al. Direct imaging of hydrogen within a crystalline environment. Appl Phys Express. 2010;3:116603.

[149] Okunishi E, Sawada H, Kondo Y. Experimental study of annular bright field (ABF) imaging using aberration-corrected scanning transmission electron microscopy (STEM). Micron. 2012;43:538.

[150] Guzmán R, Maurel L, Langenberg E, Lupini AR, Algarabel PA, Pardo ]A, et al. Polar-graded multiferroic SrMnO3 thin films. Nano Lett. 2016;16:2221.

[151] Kim YM, Pennycook S], Borisevich AY. Quantitative comparison of bright field and annular bright field imaging modes for characterization of oxygen octahedral tilts. Ultramicroscopy. 2017;181:1.

[152] Gao P, Kumamoto A, Ishikawa R, Lugg N, Shibata N, Ikuhara Y. Picometer-scale atom position analysis in annular bright-field STEM imaging. Ultramicroscopy. 2018;184:177.

[153] Zhou D, Müller-Caspary K, Sigle W, Krause FF, Rosenauer A, van Aken PA. Sample tilt effects on atom column position determination in ABF-STEM imaging. Ultramicroscopy. 2016;160:110.

[154] Choi T, Horibe Y, Yi HT, Choi Y], Wu W, Cheong SW. Insulating interlocked ferroelectric and structural antiphase domain walls in multiferroic YMnO3. Nat Mater. 2010;9:253.

[155] Kumagai Y, Spaldin NA. Structural domain walls in polar hexagonal manganites. Nat Commun. 2013;4:1540.

[156] Han MG, Zhu Y, Wu L, Aoki T, Volkov V, Wang X, et al. Ferroelectric switching dynamics of topological vortex domains in a hexagonal manganite. Adv Mater. 2013;25:2415.

[157] Zhang S, Gilbert I, Nisoli C, Chern G-W, Erickson M], O'Brien L, et al. Crystallites of magnetic charges in artificial spin ice. Nature. 2013;500:553.

[158] Zhang QH, Tan CT, Gu L, Yao Y, Jin CQ, Wang YG, et al. Topology breaking of the vortex in multiferroic Yo.67Luo.33MnO3. Appl Phys Lett. 2014;105:12902.

[159] Tian L, Wang Y, Ge B, Zhang X, Zhang Z. Direct observation of interlocked domain walls and topological four-state vortex-like domain patterns in multiferroic YMnO3 single crystal. Appl Phys Lett. 2015;106:112903.

[160] Li ], Li ], Chiang FK, Chen Z, Ma C, Chu MW, et al. Homotopy-theoretic study \& atomic-scale observation of vortex domains in hexagonal manganites. Sci Rep. 2016;6:28047.

[161] Artyukhin S, Delaney KT, Spaldin NA, Mostovoy M. Landau theory of topological defects in multiferroic hexagonal manganites. Nat Mater. 2014;13:42.

[162] Ceng Y, Lee N, Choi Y], Cheong SW, Wu W. Collective magnetism at multiferroic vortex domain walls. Nano Lett. 2012;12:6055.

[163] Das H, Wysocki AL, Ceng Y, Wu W, Fennie C]. Bulk magnetoelectricity in the hexagonal manganites and ferrites. Nat Commun. 2014;5:2998.

[164] Zhang Q, Tan C, Gu L, Yao Y, Jin C, Wang Y, et al. Direct observation of multiferroic vortex domains in YMnO3. Sci Rep. $2013 ; 3: 2741$.

[165] Holtz ME, Shapovalov K, Mundy JA, Chang CS, Yan Z, Bourret E, et al. Topological defects in hexagonal manganites: inner structure and emergent electrostatics. Nano Lett. 2017;17:5883.

[166] Zhang M, Olson EA, Twesten RD, Wen JC, Allen LH, Robertson IM, et al. In situ transmission electron microscopy studies enabled by microelectromechanical system technology. ] Mater Res. 2005;20:1802.

[167] Allard LF, Bigelow WC, Jose-Yacaman M, Nackashi DP, Damiano ], Mick SE. A new MEMS-based system for ultra-high-resolution imaging at elevated temperatures. Microsc Res Tech. 2009;72:208.

[168] Creemer JF, Santagata F, Morana B, Mele L, Alan T, lervolino E, et al. An all-in-one nanoreactor for high-resolution microscopy on nanomaterials at high pressures. Proc IEEE Int Conf Micro Electro Mech Syst. 2011;1103.

[169] Mele L, Santagata F, Pandraud G, Morana B, Tichelaar FD, Creemer JF, et al. Wafer-level assembly and sealing of a MEMS nanoreactor for in situ microscopy. ] Micromech Microeng. 2010;20:85040.

[170] Morana B, Santagata F, Mele L, Mihailović M, Pandraud G, Creemer JF, et al. A silicon carbide MEMS microhotplate for nanomaterial characterization in TEM. Proc IEEE Int Conf Micro Electro Mech Syst. 2011;380.

[171] Protochips. Available at: https://www.protochips.com/products/fusion/. Accessed 2019.

[172] DENSsolutions. Available at: https://denssolutions.com/products/wildfire/. Accessed 2019.

[173] Hummingbird. Available at: http://hummingbirdscientific.com/products/heating-biasing/. Accessed 2019. 
[174] He K, Robertson AW, Fan Y, Allen CS, Lin YC, Suenaga K, et al. Temperature dependence of the reconstruction of zigzag edges in graphene. ACS Nano. 2015;9:4786.

[175] Harmand JC, Patriarche G, Glas F, Panciera F, Florea I, Maurice ]L, et al. Atomic step flow on a nanofacet. Phys Rev Lett. 2018;121:166101.

[176] Ryu CH, France-Lanord A, Wen Y, Zhou S, Grossman JC, Warner ]H. Atomic structure and dynamics of self-limiting sub-nanometer pores in monolayer WS2. ACS Nano. 2018;12:11638.

[177] Yamamoto N, Yagi K, Honjo G. Electron microscopic studies of ferroelectric and ferroelastic Cd2(MoO4)3. IV. Polarization reversal and field induced phase transformation. Phys Status Solidi. 1980;62:657.

[178] Snoeck E, Normand L, Thorel A, Roucau C. Electron microscopy study of ferroelastic and ferroelectric domain wall motions induced by the in situ application of an electric field in BaTiO3. Phase Transitions. 1994:46:77.

[179] Randall CA, Barber D], Whatmore RW, Groves P. A TEM study of ordering in the perovskite, $\mathrm{Pb}(\mathrm{Sc1} / 2 \mathrm{Ta1} / 2)$ O3. ] Mater Sci. 1986;21:4456.

[180] Tan X, He H, Shang ]-K. In situ transmission electron microscopy studies of electric-field-induced phenomena in ferroelectrics. ] Mater Res. 2005;20:1641

[181] He H, Tan X. Electric-field-induced transformation of incommensurate modulations in antiferroelectric Pbo.99Nbo.02[(Zr1-xSnx)1yTiy]0.9803. Phys Rev B. 2005;72:024102.

[182] Qu W, Zhao X, Tan X. Evolution of nanodomains during the electric-field-induced relaxor to normal ferroelectric phase transition in a Sc-doped Pb(Mg1/3Nb2/3)O3 ceramic. ] Appl Phys. 2007;102:84101.

[183] Sato Y, Hirayama T, Ikuhara Y. Real-time direct observations of polarization reversal in a piezoelectric crystal: $\mathrm{Pb}(\mathrm{Mg} / 3 \mathrm{Nb} 2 / 3) \mathrm{O} 3-$ $\mathrm{PbTiO}_{3}$ studied via in situ electrical biasing transmission electron microscopy. Phys Rev Lett. 2011;107:187601.

[184] Nelson CT, Gao P, Jokisaari JR, Heikes C, Adamo C, Melville A, et al. Domain dynamics during ferroelectric switching. Science. 2011;334:968.

[185] Chang H, Kalinin SV, Yang S, Yu P, Bhattacharya S, Wu PP, et al. Watching domains grow: in situ studies of polarization switching by combined scanning probe and scanning transmission electron microscopy. ] Appl Phys. 2011;110:52014.

[186] Zhang JX, Xiang B, He Q, Seidel ], Zeches R], Yu P, et al. Large field-induced strains in a lead-free piezoelectric material. Nat Nanotechnol. 2011;6:98.

[187] Gao P, Britson ], Jokisaari JR, Nelson CT, Baek SH, Wang Y, et al. Atomic-scale mechanisms of ferroelastic domain-wall-mediated ferroelectric switching. Nat Commun. 2013;4:2791.

[188] Gao P, Britson ], Nelson CT, Jokisaari JR, Duan C, Trassin M, et al. Ferroelastic domain switching dynamics under electrical and mechanical excitations. Nat Commun. 2014;5:3801.

[189] Gao P, Nelson CT, Jokisaari JR, Baek SH, Bark CW, Zhang Y, et al. Revealing the role of defects in ferroelectric switching with atomic resolution. Nat Commun. 2011;2:591.

[190] Gao P, Nelson CT, Jokisaari JR, Zhang Y, Baek SH, Bark CW, et al. Direct observations of retention failure in ferroelectric memories. Adv Mater. 2012;24:1106.

[191] Chen L, Ouyang ], Ganpule CS, Nagarajan V, Ramesh R, Roytburd AL. Formation of $90^{\circ}$ elastic domains during local $180^{\circ}$ switching in epitaxial ferroelectric thin films. Appl Phys Lett. 2004;84:254.

[192] Lu H, Bark CW, Esque De Los Ojos D, Alcala ], Eom CB, Catalan C, et al. Mechanical writing of ferroelectric polarization. Science. 2012;336:59.

[193] Han MG, Marshall MS, Wu L, Schofield MA, Aoki T, Twesten R, et al. Interface-induced nonswitchable domains in ferroelectric thin films. Nat Commun. 2014;5:4693.

[194] Lee ]K, Shin CY, Song K, Choi WS, Shin YA, Park SY, et al. Direct observation of asymmetric domain wall motion in a ferroelectric capacitor. Acta Mater. 2013;61:6765.

[195] Scott JF. Applications of modern ferroelectrics. Science. 2007;315:954.

[196] Zhang DL, Huang WC, Chen ZW, Zhao WB, Feng L, Li M, et al. Structure evolution and multiferroic properties in cobalt doped Bi4NdTi3Fe1-xCoxO15-Bi3NdTi2Fe1-xCoxO12-ס intergrowth Aurivillius compounds. Sci Rep. 2017;7:43540. 